\title{
MULTIELEMENT FLAME EMISSION SPECTROSCOPY USING A SCANNING FABRY-PEROT \\ INTERFEROMETER
}

\author{
Gary Albert Korba
}

M. S. Thesis Submittcd to Iowa State University

\author{
Ames Laboratory, DOE \\ Iowa State University \\ Ames, Iowa 50011
}

Date Transmitted: April 1978

PREPARED FOR THE U. S. DEPARTMENT OF ENERGY UNDER. CONTRACT NO. W-7405-eng-82

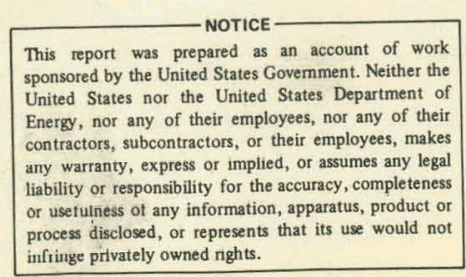




\section{DISCLAIMER}

This report was prepared as an account of work sponsored by an agency of the United States Government. Neither the United States Government nor any agency Thereof, nor any of their employees, makes any warranty, express or implied, or assumes any legal liability or responsibility for the accuracy, completeness, or usefulness of any information, apparatus, product, or process disclosed, or represents that its use would not infringe privately owned rights. Reference herein to any specific commercial product, process, or service by trade name, trademark, manufacturer, or otherwise does not necessarily constitute or imply its endorsement, recommendation, or favoring by the United States Government or any agency thereof. The views and opinions of authors expressed herein do not necessarily state or reflect those of the United States Government or any agency thereof. 


\section{DISCLAIMER}

Portions of this document may be illegible in electronic image products. Images are produced from the best available original document. 


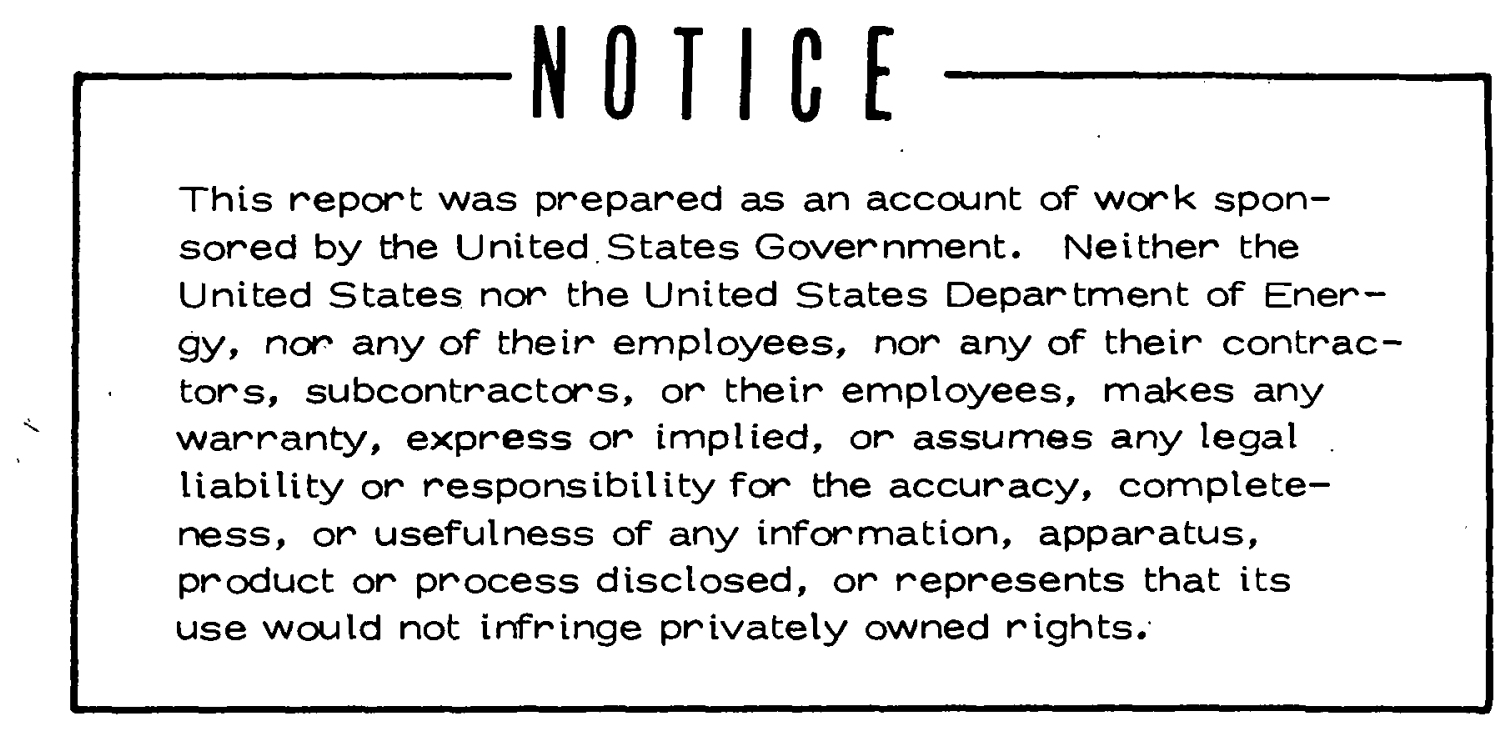

Available from: National Technical Information Service

U. S. Department of Commerce

P.O. Box 1553

Springfield, VA 22161
Price: Microfiche $\$ 3.00$
Paper Copy $\$ 5.00$




\begin{abstract}
Multielement flame emission spectroscopy using
a scanning Fabry-Perot interferometer
\end{abstract}

by

Gary Albert Korba

\begin{abstract}
A Thesis Submitted to the
Graduate Faculty in Partial Fulfillment of

The Requirements for the Degree of

MASTER OF SCIENCE
\end{abstract}

Department: Chemistry

Major: Analytical Chemistry

Approved :

$\frac{\text { E..S. Uamy. }}{\text { In charge of Major work }}$

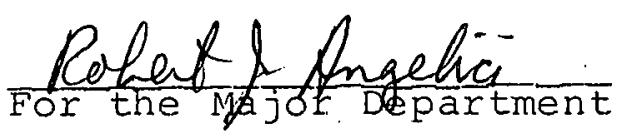

$\frac{\text { For the Graduate college }}{\text { Tous }}$

Iowa state University

Ames, Iowa 
TABLE OF CONTENTS

Page

ABSTRACT

$i v$

I. INTRODUCTION 1

II. LITERATURE REVIEW ' 4

A. Flame Emission Spectroscopy 4

B. Detection Systems for Multielement Spectros copy

C. Fabry-Perot Interferometry 12

$\begin{array}{ll}\text { III. INSTRUMENTATION } & 17\end{array}$

A. Nebulizer-Burner System $\quad 17$

B. Optical system 18

C. Detection System . 21

D. Computer Interface 24

IV. EXPERIMENTAL RESULTS AND DISCUSSION 29

A. Single Element Analysis 29

B. Multielement Analysis . 41

1. Tap water 46

2. Urine and serum. 49

3. Orchard leaves 51

4. Steel 54

V. SUMMARY $\quad 58$

VI. SUGGESTIONS FOR FUTURE STUDY . . 60

$\begin{array}{lll}\text { VII. BIBLIOGRAPHY } & 62\end{array}$ 
VIII. ACKNOWLEDGMENTS

IX. APPENDIX A: ELECIRONIC CIRCUITRY

A. Ramp Generator

B. High Voltage Amplifier

C. Buffer/Inverter

D. Sample-Hold Detection System 


\begin{abstract}
A system for multielement flame emission analysis based on a scanning Fabry-Perot interferometer is described and evaluated. Detection limits and linearity of response for ten elements commonly determined by flame photometry compare favorably to conventional single-element methods. Analyses for several elements in tap water, serum, urine, NBS SRM 1571 orchard leaves and low alloy steel demonstrate the excellent accuracy and precision of the technique. Resolution of the system allows up to five elements to be determined simultaneously.
\end{abstract}




\section{INTRODUCTION}

The important role that small amounts of various elements play in physical, chemical and biological systems has emerged as methods of analysis have increased in sensitivity and utility. Although a great deal of progress in elemental analysis may be attributed to the demands that arise out of other areas of research, many of the recent advances are also a result of the rapid development of new and improved instrumentation involving widely differing principles and capabilities. In terms of the amount of information obtained in a given analysis, the various methods available are most conveniently classified as either single or multielement techniques. A single-element method is optimized to determine the particular element with as high an accuracy and precision as possible whereas most multielement methods must involve a compromise in operating conditions to encompass the different behaviors of the various elements in a given sample.

The main impetus behind the development of multielement methods of analysis stems from the demand for the capability to determine several elements in a single sample in a simple, rapid, reliable and inexpensive way. There are many analytical problems e.g., clinical, metallurgical and geochemical, in which the application of such multielement 
methods is particularly important. Unlike atomic absorption and fluorescence methods which generally require one light source for each element or small group of elements determined, atomic emission ( $A E$ ) spectroscopy lends itself most readily to multielement analysis because the processes of atomization and excitation are accomplished using the same source regardless of which elements are being determined.

In multielement $A E$ spectroscopy, the objective is to record one or more components of the ultraviolet-visible spectrum for each of several species being analyzed during a single sampling and atomization process. To achieve this goal, rapid single channel or multichannel devices are necessary. Historically, AE multichannel systems have enjoyed the greatest popularity with the photographic and multiple-slit-multiple-detector (direct reader) types of instruments. Howevex, both of these systems leave much to be desired. The interpretation and quantitation of photographic spectra is tedious and suffers from poor accuracy and precision, while the direct reading spectrometers require as many detectors as elements to be determined and are often difficult to align for closely spaccd emission lines. The high resolution available with modern scanning Fabry-Perot (FP) interferometers makes them a potential alternative to the conventional dispersion optics in multielement 
spectrometers. Moreover, FP interferometers are now available which possess not only good resolution but also excellent throughput and a large enough free spectral range such that overlapping orders of different emission lines may often be avoided. In a preliminary investigation, Pruiksma, Ziemer and Yeung (1) proposed the use of a scanning FP interferometer for multielement atomic emission analysis. This initial work demonstrated that such a system might be feasible if the proper design parameters could be developed. The purpose of this thesis is to examine the full potential of multielement flame emission analysis using a scanning FP interferometer as a wavelength selection device. Research to this end included the dcsign and construction of a low-cost electronic detection system as well as a minicomputer interface which was used for signal-averaging .. purposes. Detection limits for ten elements which are commonly determined by flame spectroscopic methods and which have sensitive emission lines in the visible spectral region are presented. Several analyses on steel, serum, urine, tap water and NBS SRM 1571 orchard leaves were performed to demonstrate the excellent accuracy and precision of the technique. Finally, a discussion of the system's inherent advantages and disadvantages as well as future applications are presented. 


\section{LITERATURE REVIEW}

\section{A. Flame Emission Spectroscopy}

With the increasing development of instrumental methods of analysis, analysts are confronted with the task of selecting a suitable technique to solve their problems. There are several methods of multielement analysis available, which vary widely in their application and scope. These methods include: spark source mass spectrometry (2), neutron activation (3-5), x-ray fluorescence (6-8), electrochemical techniques $(9,10)$, and optical atomic spectroscopy. The last one is by far the most popular for the solution of a wide variety of problems due to its high precision and accuracy, simplicity, reasonable sensitivity and relatively low cost. As mentioned previously AE spectroscopy is the best choice for multielement optical spectroscopic analysis. In atomic emission spectra, atoms which have been excited from a ground state to a higher energy level return to a lower level by liberating energy corresponding to a frequency $v$ given by the well-known equation $\Delta E=h v$. This process occurs according to transitions governed by selection rules (11, 12). The fraction of atoms excited depends exponentially on the excitation source temperature. If the source is considered to be in a state of thermal equilibrium, then the relationship of 
the temperature to the distribution of atoms of the analyte with respect to energy levels can be determined by the Boltzmann distribution law (13). The intensity of the emitted radiation depends on the probability of the transition from the excited state to a state of lower energy, the number of neutral atoms and the fraction of the population of atoms which is excited. Condon and Shortley (14) and Herzberg (15) have written excellent texts on the theory and historical development of atomic spectra. Atomic energy level diagrams for elements determinable by atomic emission spectroscopy have been published by Mavrodineanu and Boiteux (16). The total intensity of thermal radiation of metal spectra has been discussed comprehensively by winefordner et al. (17), including expressions for the total intensity of emission from atoms at low and high concentrations.

Various methods of excitation for AE spectroscopy exist. The dc arc and spark are both commonly known methods and their advantageous features and limitations are discussed extensively and clearly in a number of books (18-20). The dc plasma jet (21) has been found to give fair detection limits (22) for a large number of elements but it is plagued with disadvantages such as high background, ionization interferences and considerable consumption of gases and 
erosion of the electrodes. The inductively coupled, radiofrequency plasma $(23,24)$ offers an optically thin, high temperature source which is nicely suited for AE spectroscopy. Its performance is very good and has been well documented $(25,26)$, with emission from virtually all elements having been recorded (24). However, ionization interferences are greater and must be carefully investigated in order to be minimized. Although the rf plasma might be the best source for multielement AE spectroscopy, it is also the most complicated and expensive, requiring elaborate power supplies, rf generators and impedance matching networks between the generator and load coils. Thus, the flame represents, in most cases, a satisfactory excitation source due to its simplicity, safety, ease of operation and stability.

Although photographic spectra of the sun were taken around 1840 following the production of silver chloride photographic papers, no significant progress in flame spectroscopy came until 1860 when Kirchhoff and Bunsen (27) definitely correlated visible emission lines with specific elements, This discovery immediately pointed the way to chemical analysis by spectral observation of the flame and within ten years, attempts at quantitative flame emission analysis were being made by Janssen (28). However, it is necessary to recognize that the first widespread presentation of 
emission flame photometry, as it is conceived of today, was the paper by Barnes, Richardson, Berry and Hood (29) in 1945 . By 1960, the first commercial flame photometers had appeared and analytical chemists began to use and accept flame photometry as a reliable instrumental analytical method for quantitative analysis. In 1955 the first book in the world specifically devoted to flame photometry was authored by Burriel-Marti and Ramírez-Muñoz (30), in precisely the same year that the other main branch of flame analysis, atomic absorption, was announced as a new possibility in analytical work. Since that time several excellent texts have been written on flame emission spectrochemical analysis $(16,31,32,33,34)$. Numerous niethods employing the direct transfer of the analyte into the flame have been attempted with the aim of circumventing the problems posed by the dissolution of the sample prior to analysis. These include generation of metal particles (35) and the direct atomization of suspensions (36). However, in the great majority of cases the sample to be atomized is brought into solution and then aspirated into the flame by means of a pneumatic nebulizor. Gonerally; flames of the type hydrogen-oxygen, acetylene-oxygen or acetylene-nitrous oxide are employed. The thermal equilibria existing in hydrogenoxygen. and acetylene-oxygen flames as well as in air-hydrogen and argon-hyjdrogen-entrained air flames have been discussed 
by de Gaian and winefordner (37). With conventional single element flame emission, 44 elements have been determined at concentrations of less than $1 \mu \mathrm{g} / \mathrm{mL}$ (38).

To list all the analytical problems to which flame emission spectroscopy has been applied would be next to impossible. It has been applied to the determination of virtually every element in the periodic table in almost every imaginable matrix, primarily at trace or low concentration levels. Mavrodineanu (39) has thoroughly reviewed the analytical applications of flame spectroscopy prior to 1966 . As more and more is understood about mineral metabolism, the routine monitoring of trace elements in water, soils, plants, urine, blood and other biological tissues has also become important $(40,41,42)$. Since samples of this type are many times available in only small amounts, multielement $A E$ spectroscopy becomes a valuable analytical tool for the analysis of such materials. As a matter of fact, flame emission analysis is the recommended analytical procedure for $N a, K$ and $L i$ in serum and urine $(44,45)$ while Berman (4.3) describes conditions for the analysis of nine metals in a variety of biological samples by flame emission spectroscopy. Other interesting applications of $\mathrm{AE}$ spectrometry, where multielement analysis would be useful include: analysis of geological materials $(34,46,47)$, coal (48), petroleum products (49) and alloys (50). 
B. Detection systems for Multielement spectroscopy

To perform multielement spectrometry, a device capable of measuring intensities at different wavelengths is necessary, i.e., a multichannel dovice is needed. An extensive review of multielement spectroscopic techniques has recently been published by winefordner, Fitzgerald and Omenetto (51). Basically, multichannel detection systems can be divided into two major categories: temporal and spatial.

Temporal devices rely upon one single detector. The spectral information is encoded in the time domain and thus the multielement information collected is sequential. A variety of spectral scanning systems have been developed for temporal multichannel detection $(52,53)$. These systems are based on moving the detector if the dispersion optics are held stationary (54) or scanning the spectrum past a fixed slit either by oscillating the dispersing element (55) or oscillating an auxiliary mirror (56). Santini, Milano and Pardue (57) and Pimentel (58) have written excellent reviews on various rapid scanning technqiues. Dawson, Ellis and Milner (59) describe a system capable of monochromator scan rates of up to $1800 \mathrm{~nm} / \mathrm{scc}$ however, geometrical distortions arise when optical components are rotated at these high speeds and hence poor signal-tonoise ratios have resulted for the low light fluxes present 
in AE spectrometry. The most important consideration then becomes the design of a rapid-scanning device capable of determining the wavelength being passed by the exit slit of the spectrometer at any instant while at the same time accurately reproducing that wavelength from scan to scan. Cordos and Malmstadt. (60), and Johnson, Plankey and Winefordner (61) have used a computer to control the monochromator such that it will slew between wavelengths of analytical interest and remain at a given wavelength until the rest of the system has been optimized for that particular element. Typically 2 to 5 seconds is required to optimize conditions for each measurement step.

Another approach to temporal multielement analysis simply employs a rotating filter wheel in place of a monochrometer. Several papers have been published describing the advantages of such filter-detection systems $(62,63$, 64). Generally, the disadvantages and problems encountered with this type of system are high background and insufficient spectral discrimination, and thus it does not readily lend. itself to trace multielement analysis.

Spatial devices have traditionally been the most popular multielement detection systems for AE spectrometry. They rely upon multiple detectors and hence information from more than one spectral element is acquired simultaneously 
and independently. The first and perhaps most widely used multichannel detector has been the photographic plate (65, 66, 67). Among the limitations of photographic detection are low efficiency, limited dynamic range, nonlinearity and most importantly, the length of time involved in acquiring and processing the data.

Direct-reading spectrometers have been developed in which selected spectral lines of interest are isolated from the dispersed radiation by exit slits positioned along the focal plane of the spectrometer and detected by photomultipliers positioned behind each slit. This approach was first used for simultaneous flame emission analysis of $\mathrm{Na}$, $\mathrm{K}$, Ca and Sr by Vallee and Margoshes (68) in 1956 and since then by many others $(69,70,71,72,73)$. In addition, several manufacturers produce direct-reading spectrometers for use with argon inductively coupled plasmas (Jarrell-Ash JY 38 P spectroanalyzer), dc arc plasma jets (spectrametrics SpectraJet II), and for clinical flame photometry (Radiometer Model FLM 3), Since the biggest problem with using a direct-reading spectrometer is the positioning and alignment of the exit slits at the proper point on the focal plane, it cannot be conveniently changed to monitor other elements. Scanning methods employing vibrating slits or quartz plates have been used with some success for background 
correction $(74,75)$ while Haisch, Laqua and Hagenah used glass fiber optics to simultaneously measure adjacent wavelengths (76).

In 1970, Margoshes (77) reported that the television tube would seem to be an ideal detector for multielement determinations since, in principle, it combines the advantages of photographic detection with those of photomultiplier tubes. Actually, the first application of television tubes to atomic spectrometry was by Benn, Foote and Chase (78) who in 1949 used an image orthicon to examine low-intensity emission lines during a gaseous combustion reaction. Several papers have recently appeared on the use of vidicons $(79,80,81)$ and photodiode arrays $(82,83)$ as well as new types of semiconductor devices such as charge coupling (84) and charge injection (85) detectors for multielement atomic spectroscopy. Although these image devices are indeed attractive, considerable refinement and development is still warranted if their performance is to approach that of photomultiplier tubes.

\section{Fabry-Perot Interferometry}

The: interferometer devised by C. Fabry and A. Perot in 1897 (86) employs the principles of multiple beam interference. The superior luminosity obtainable with modern FP 
interferometers has been shown by Jacquinot (87) and they have been used for measuring the wavelength stability of lasers (88), examining hyperfine structure (89), isotopic analysis (90), rapid wavelength scanning (91) line profile measurements (92) and in conjunction with normal monochromators for high resolution atomic absorption spectrometry (93).

The first to recognize the importance of multiple beam interference was Bouluuch who, in a brief notc in 1893, gave the theory of formation of a fringe system which shortly afterwords was incorporated into the FP interferometer (94). Several authors $(94,95,96,97)$ have provided excellent discussions on multiple beam interference and its application to FP interferometers.

A FP cavity is constructed using two flat optical plates that are parallel to one another with partially transmitting coatings on their inner surfaces and antireflection coatings on their outside surfaces. Normally, the outside surfaces are wedged slightly with respect to the inside surface to avoid forming additional cavities. FP interferometers typically use 15 to $50 \mathrm{~mm}$ diameter mirrors with flatnesses of $\lambda / 50$ to $\lambda / 200$ (normally specified for $\lambda=500 \mathrm{~nm}$ ). Multilayer dielectric coatings are available which are applicable over the entire visible region of the spectrum (98). If monochromatic radiation of wavelength $\lambda$ is incident 
upon a FP cavity, it will be transmitted and undergo maximum constructive interference when

$$
\mathrm{m} \lambda=2 n d \cos \theta
$$

where $\mathrm{m}$ is the order of interference (an integer), $n$ is the refractive index of the medium between the two reflecting surfaces, $d$ is the mirror spacing and $\theta$ is the inclination of the normal of the mirrors to the incident wavefront direction (99). It should be noted that each change of $\lambda / 2$ in the product nd is equivalent to a change in $m$ of unity and therefore successive changes result in repetitive scans of the same free spectral range (FSR). The FSR is defined as the separation between simultaneously transmitted wavelengths and is given by

$$
\operatorname{FSR}=\lambda^{2} / 2 n d
$$

and thus, the larger the FSR, the less chance there is for two adjacent wavelengths to be transmitted by the FP at any given mirror spacing.

The measure of the FP interferometer's ability to resolve closely spaced. lines is called the finesse. The two factors which limit the finesse most are reflection of less than unity by the mirrors and the lack of parallelism or flatness of the mirrors (99). For a fixed mirror flatness, the reflective finesse $\left(F_{R}\right)$ is calculated using 


$$
\mathrm{F}_{\mathrm{R}} \doteq \pi \sqrt{\mathrm{R} / 1-\mathrm{R}}
$$

where $R$ is the reflectivity of the mirrors. If $A$ and $T$ are the fraction of the incident energy absorbed and transmitted by the mirrors respectivelv, then the maximum transmission of the interferometer $\left(t_{\max }\right)$ is given as

$$
t_{\max }=\left(\frac{1-A-R}{1-R}\right)^{2}
$$

Thus if $A$ is zero, the peak intensity of the transmitted fringes is equal to the intensity of the incident light even for values of $R$ close to unity and so, if $A$ is small enough it is possible to have both excellent throughput and resolution with the same interferometer (100).

The scanning FP interferometer has been known since 1948 when Jacquinot and Dufour (101) demonstrated the method of pressure scanning. The two most common methods of varying the product nd in Equation (I) are changing the pressure of the air between the two mirrors (changing $n$ ) (102), or displacing one of the mirrors by applying a linear voltage ramp to a piezoelectric crystal (changing d) (103). Piezoelectric scanning is the most versatile and convenient method of wavelength scanring (104).

The application of a scanning FP interferometer to multielement flame emission analysis now becomes obvious: if the atomic emission from several elements in a flame is 
directed through a FP cavity with a photoelectric detector placed behind it, it would be possible to record the emission intensities of all elements present as the mirrors are scanned at least far enough so that one order of each line will be observed. 


\section{INSTRUMENTATION}

A. Nebulizer-Burner system

Hydrocarbon flames are the most widely used for atomic emission spectrometry (33) however, an oxyhydrogen flame was chosen for this work because of its extremely low background radiation. Although the temperature of this flame is somewhat lower than the corresponding acetylene one (16), none of the elements considered here had high enough excitation energies to warrant the use of the more luminous hydrocarbon flames. A total-consumption nebulizer-burner system was used throughout the entire study nrimarily because of the ease and safety with which the $\mathrm{H}_{2} / \mathrm{O}_{2}$ flame can be handled. With this type of system, the functions of nebulizer and burner are combined in the same unit. A capillary for the solution intake is positioned concentrically inside the nozzle for the oxidant gas (oxygen). The latter nozzle is, in turn, placed concentrically in a somewhat wider, separate nozzle for the fuel gas (hydrogen). The outlets of these nozzles thus form the tip of the burner; no mixing of fuel gas and oxidant gas takes place before they leave the burner. The compressed $\mathrm{O}_{2}$ flow aspirates and nebulizes the liquid. Turbulent mixing of the $\mathrm{O}_{2}$ flow and spray with the fuel gas is achieved above the burner tip and, hence, combustion takes place at some distance from this tip, where the mixing has proceeded 
sufficiently far.

The burner system used for this work consisted of a Beckman Model 4030 total-consumption burner with a Model 9220 Regulator. Gas flow rates are monitored using Brooks Model 1355-OOAIFAA rotameters capable of indicating flow rates of zero to $15 \mathrm{~L} / \mathrm{min}$. Following initial pressure reduction to $15 \mathrm{psi}$ by two-stage regulators on the supply cylinders, the hydrogen and oxygen pressures are reduced to 1.5 and 8.5 psi respectively using the Beckman Regulator. At these pressures, the hydrogen and oxygen flow rates correspond to 8.2 and $2.5 \mathrm{~L} / \mathrm{min}$ and resulted in an analyte solution consumption rate of $.72 \mathrm{~mL} / \mathrm{min}$ for this slightly reducing flame. The burner assembly was easily cleaned by soaking it in.005 $\mathrm{N}^{\mathrm{HNO}}{ }_{3}$ overnight.

\section{B. Optical System}

A schematic of the optical arrangement used for analysis is shown in Figure 1. Emission from the flame is collected using a quartz aspheric condenser (LI) which has a focal length of $68 \mathrm{~mm}$. The aspheric lens is designed to have a much shorter focal length than is possible with a spherical glass lens of equal diameter and hence a greater fraction of the emission intensity from the flame is directed through the $4.0 \mathrm{~mm}$ pinhole which serves as the entrance pupil to the interferometer. The radiation passing through the 


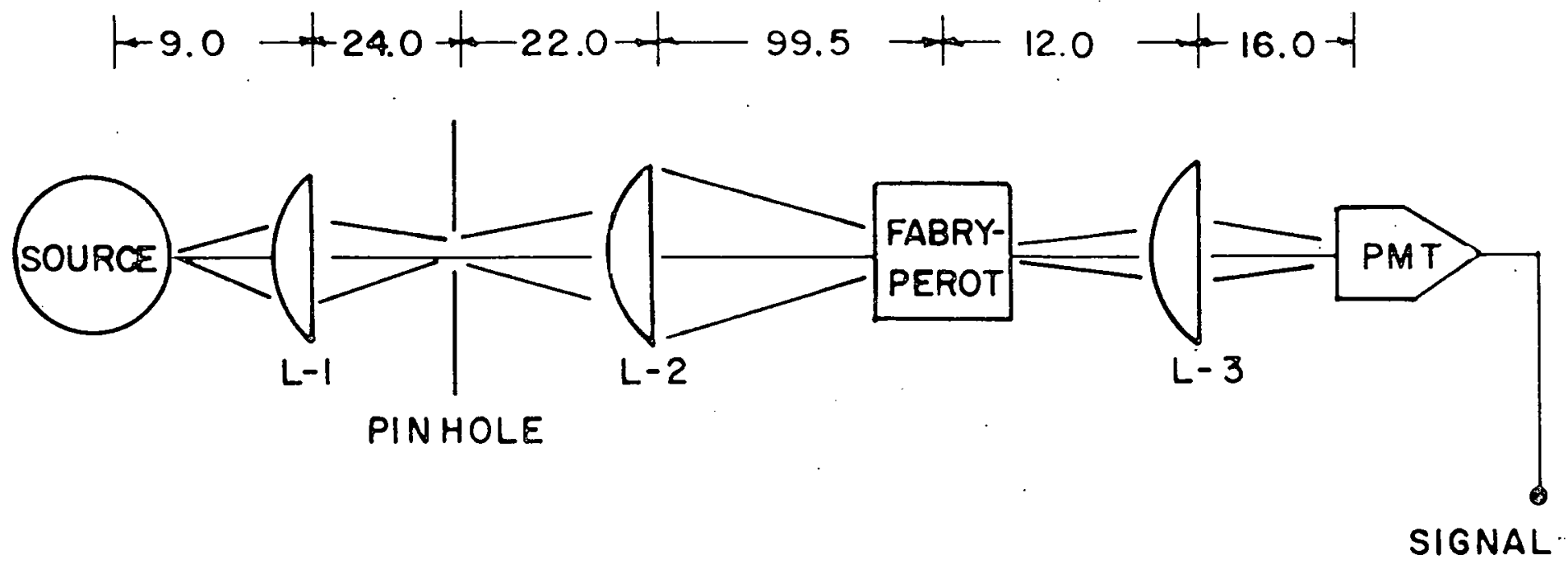

Figure 1. Optical arrangement for use with the scanning FP interferometer (spacing between components are given in $\mathrm{cm}$ ) 
pinhole is collected and focused at an angle of $\theta=18.5^{\circ}$ (see Equation 1) onto the aperture of the interferometer using a plano-convex optical crown glass lens (L2) having a 200-mm focal length.

The heart of the system is a Tropel CL-100 scanning Fabry-Perot interferometer. The cavity spacing of the etalon is adjustable from .005 to $1.0 \mathrm{~cm}$ and can be aligned mechanically via micrometers and electronically using piezoelectric crystals. Scanning is accomplished with a piezoelectric drive having a movement of $.75 \mu / 300 \mathrm{~V}$ and will respond linearly at scan rates of up to $1 \mathrm{KHz}$. The mirrors are coated for operation between 380 and $800 \mathrm{~nm}$ with a finesse of 50 or better throughout this entire region. The very small cone of light incident upon the Fabry-Perot from the plano-convex lens illuminates only the very center of the mirrors where the flatness and coatings are the most uniform, thus serving to enhance the resolution of the system as much as possible. The light focused upon the FP is not collimated (i.e. not all of the rays contained in the cone of light are at the same $\theta$ in Equation 1) and so the width of a transmitted emission line seen by the detector as the FP is scanned will be considerably greater than the atomic line width. The best resolution will be realized when the incident light is perfectly collimated. Collimation can be 
achieved using a smaller pinhole and two additional lenses (1) but this in turn results in a decrease in spectral throughput. The optical arrangement presented here represents a compromise that provides excellent sensitivity with resolution adequate to determine at least four elements simultaneously. Since the transmitted radiation emerging from the back mirror of the FP is diverging, another planoconvex lens (L3) with a focal length of $150 \mathrm{~mm}$ is used to focus the light to a photomultiplier tube for detection. Initial alignment of the system was accomplished by defining an optical axis using a helium-neon laser and then properly positioning the components along this axis. Finetuning and alignment of the Fabry-Perot is done using a high pressure sodium lamp in place of the flame. By simultaneously scanning the mirrors and monitoring the photomultiplier output on an oscilloscope the doublet structure of the sodium emission can be used to optimize the alignment by proper adjustment of the micrometers. Minor adjustment is usually required about once every one to two weeks.

\section{Detection System}

A block diagram of the associated electronics used for multielement detection is shown in Figure 2. A Topel PZM three-channel high voltage power supply is used for fine 


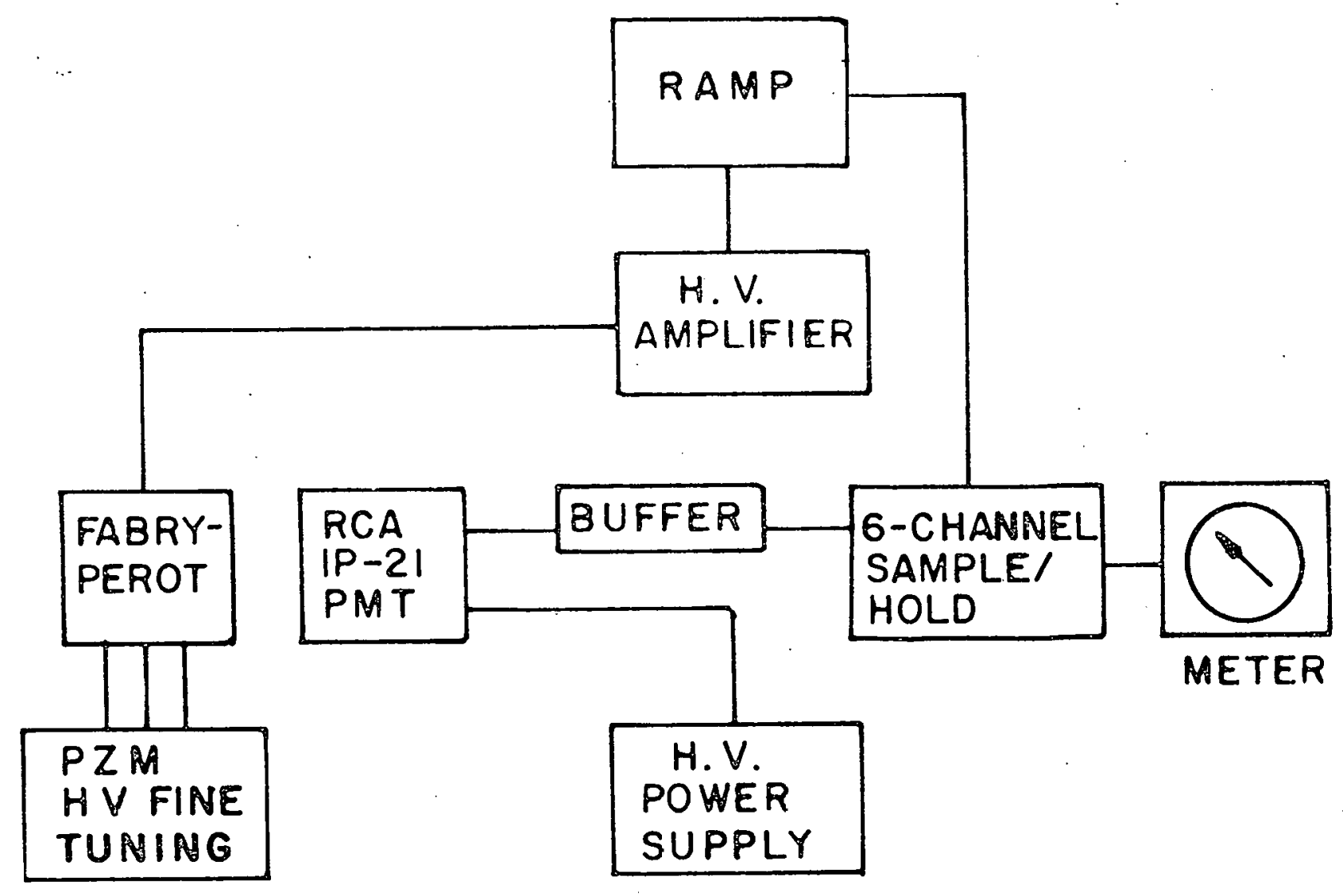

Figure 2. Block diagram of electronic components used for multielement detection 
alignment of the interferometer cavity. Each channel is in series with one of the three piezoelectric tuning crystals on the Fabry-Perot and moves $4 \mu / 100 \mathrm{~V}$.

The transmitted radiation from the Fabry-perot is detected with a 1-1/8" diameter side-on type RCA IP-21 photomultiplier tube which shows excellent response from 320 to $620 \mathrm{~nm}(105)$. The photomultiplier cathode is held negative with respect to the anode using a Hamner NV-13 high voltage power supply. Typically, the potential between the cathode and anode is 800 to $1000 \mathrm{~V}$. The output from the photomultiplier is then sent through a buffer/inverter before being sent to the detection system.

A linear ramp generator was constructed, which provides a 0 to lov-ramp with a continuously adjustable duration of $1 \mathrm{msec}$ to $10 \mathrm{sec}$ and is capable of operating in a continuous or externally retriggerable mode. The output from this ramp generator is both amplified by a factor of 30 to provide a 0 to $297-\mathrm{V}$ ramp to the scanning crystal on the interferometer and sent directly to the detection system.

The six-channel detection system designed for this work allows one to independently set each channel to any position along the ramp (i.e. to select any mirror spacing desired) and at that point to sample and hold the photomultiplier voltage. Thus, peak heights for up to six analytical lines 
may be recorded in a single scan using only one detector. The detection system makes use of a monolithic sample-hold integrated circuit which utilizes an external capacitor to hold the voltage value sampled. This feature is advantageous when the particular peak being recorded is noisy (i.e. it has some higher frequency fluctuations of a lesser magnitude superimposed upon it), because then repetitive rapid scanning will allow the capacitor to serve as a passive filter and thus decrease these higher frequency fluctuations to stabilize the signal. Sampled values may be sequentially read on an analog voltmeter as long as 5 minutes after being taken. Detailed circuit schematics for the ramp generator, high voltage amplifier, buffer/inverter and detection system may be found in Appendix A.

\section{Computer Interface}

An order to determine the ultimate detection limits for the system, the data acquisition process was interfaced to a minicomputer. Several texts discussing the principles and techniques of using a minicomputer in the laboratory have recently appeared including one by cooper (106). which specifically uses a PDP-I 1 in its examples. A block diagram of the various components involved in the interface is shown in Figure 3. Enclosed within the dashed line are the 


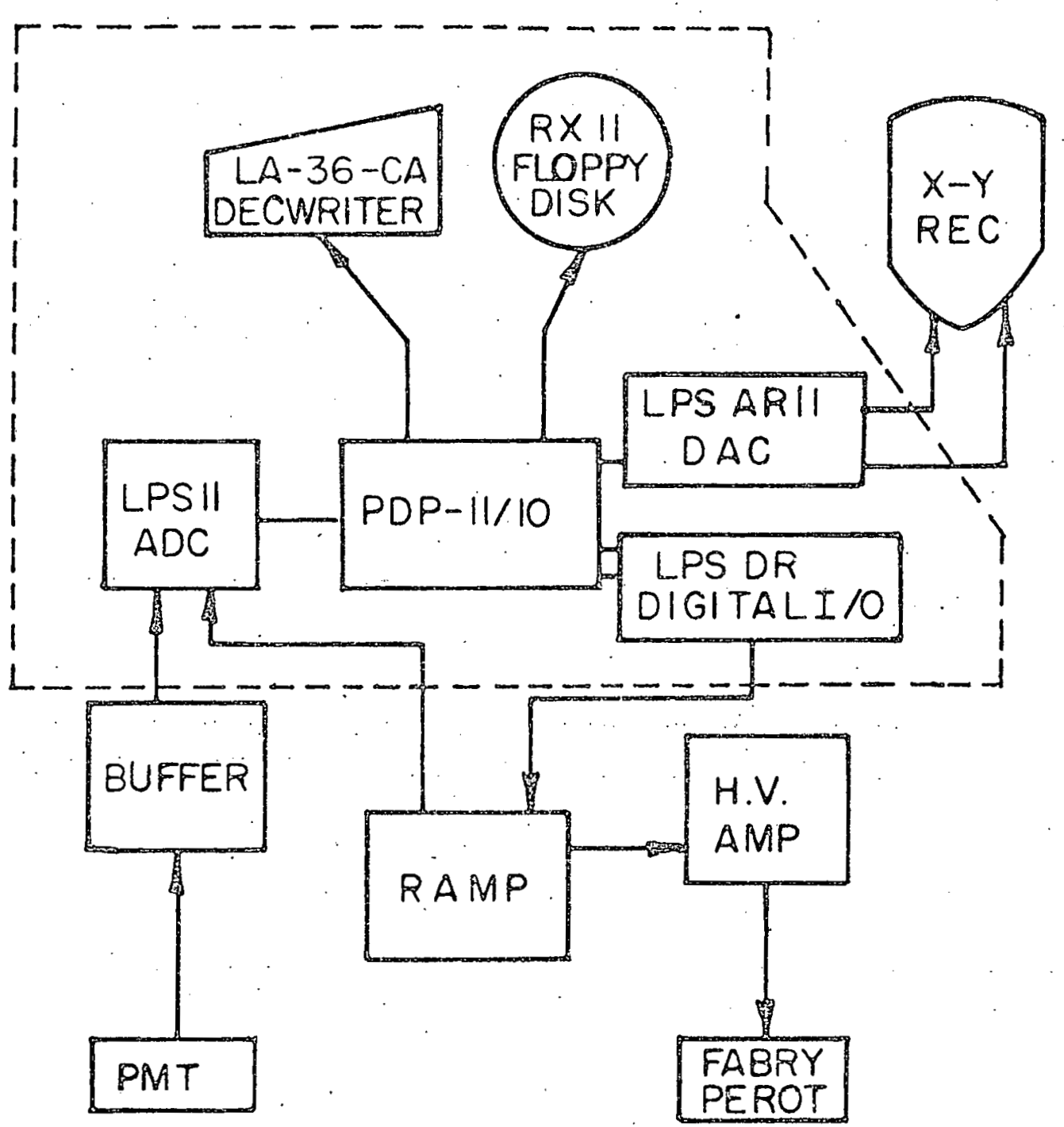

Figure 3. Interface for automated data acquisition (components enclosed within the dashed line are part of the PDP. 11/10 minicomputer system) 
devices which were inherent to the particular PDP-11/10 minicomputer available in this laboratory. They include an analog-to-digital. converter ( $A D C)$, digital-to-analog converter (DAC), digital input/output register (DI/0), dual drive floppy disk storage, Decwriter teletype terminal and $16 \mathrm{~K}$ of memory. In this work, the interface allowed for the numerical averaging of many scans (usually 1,000 to 2,000) in a small amount of time (typically $2-4$ seconds) thus allowing a considerable enhancement in the signal-to-noise ratio without the consumption of a large amount of analyte.

A simplified flowchart for the software portion of the interface is shown in Figure 4. All of the software is written in FORTRAN IV except for the data collection routine which is in the PDP-11 MACRO assembly language. After the duration and number of scans to be taken arc cntered, each scan is triggered by a TTL pulse from the DI/O register to the ramp generator. Eight hundred data points are taken on each scan with the photomultiplier output being summed to an array that is indexed according to the ramp voltage (mirror separation) at that point. Resolution by the ADC in sampling both the ramp and photomultiplier voltages is 1 part in 4096. Each time a point is summed to the array, a corresponding flag array position is incremented such that when the data acquisition is complete, each point may be properly weighted. The program then offers options for a X-Y plot of 


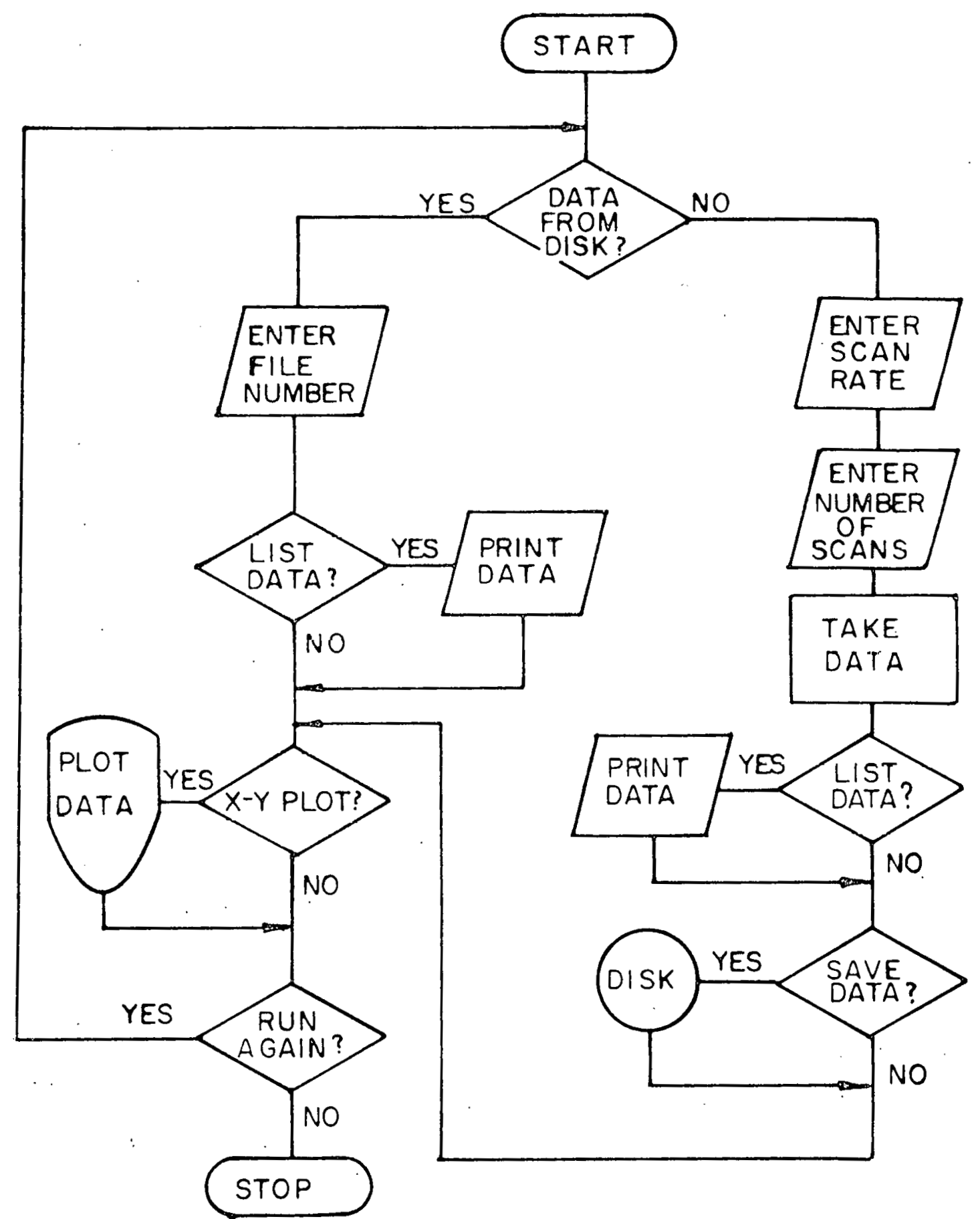

Figure 4. Flowchart for interface program (all software is written in FORTRAN TV except for the assembly language data-acquisition routine) 
the averaged spectrum, storage of the data on the floppy disk, the opportunity to graph data taken previously and to take a new spectrum. A complete listing of the software composed for this interface may be found in Appendix B. 


\section{EXPERIMENTAL RESULTS AND DISCUSSION}

\section{A. Single Element Analysis}

Before multielement data could be properly interpreted, results on individual elements had to be tabulated. Using an oscilloscope in place of the sample-hold detection system, signal traces for ten elements having visible emission lines were photographed and are shown in Figures 5-8. Each photograph of PMT response vs. mirror separation represents ten superimposed scans and demonstrates the excellent trace-to-trace repeatability of the scanning technique. All were recorded using a $10 \mathrm{msec}$ scan duration with initial and final mirror spacings of 14.8 and $15.5 \mu$ respectively. Using Equation 2, this cavity spacing gives a free spectral range of $11.6 \mathrm{~nm}$ calculated at $\lambda=589 \mathrm{~nm}$. The gain on the high voltage amplifier was set such that an entire. $74 \mu$ scan is completed in the first 7 of each $10 \mathrm{~cm}$ trace shown in the photographs. Thus in Figures $6 \mathrm{~b}$ and 8 the apparent shoulder extending for the last 3 divisions on the right-hand side of the trace is due to the fact that the maximum mirror spacing for the scan corresponded to a point where an atomic line was being transmitted. The photographs were taken over a period of several weeks and although the mirror spacings were not grossly altered, periodic fine-tuning (which does slightly change the mirror spacing) was performed and therefore the 


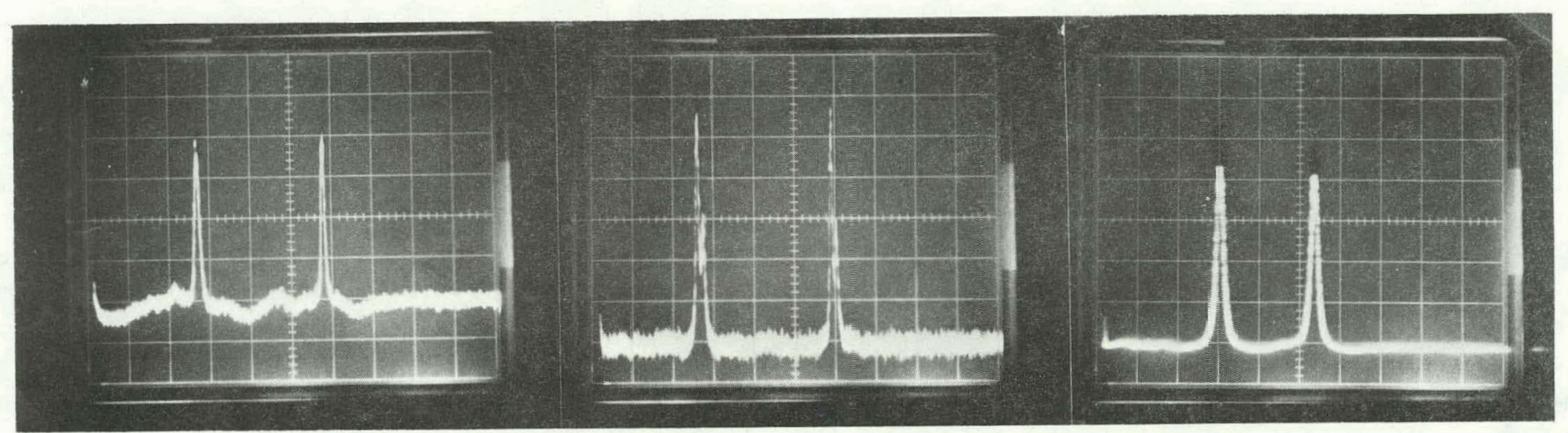

Figure 5. Signal traces for $90 \mathrm{ppm} \mathrm{Ba} \mathrm{(a),} \mathrm{.06} \mathrm{ppm} \mathrm{Na} \mathrm{(b)} \mathrm{and} 5 \mathrm{ppm} \mathrm{Ca} \mathrm{(c).}$ Peaks and order of interference $(\mathrm{m})$ from left to right are:

(a) Ba $553.6 \mathrm{~nm}(\mathrm{~m}=51,52)$, (b) Na doublet $589 \mathrm{~nm}(\mathrm{~m}=48,49)$, (c) $\mathrm{Ca} 422.7 \mathrm{~nm}(\mathrm{~m}=67,68)$ 


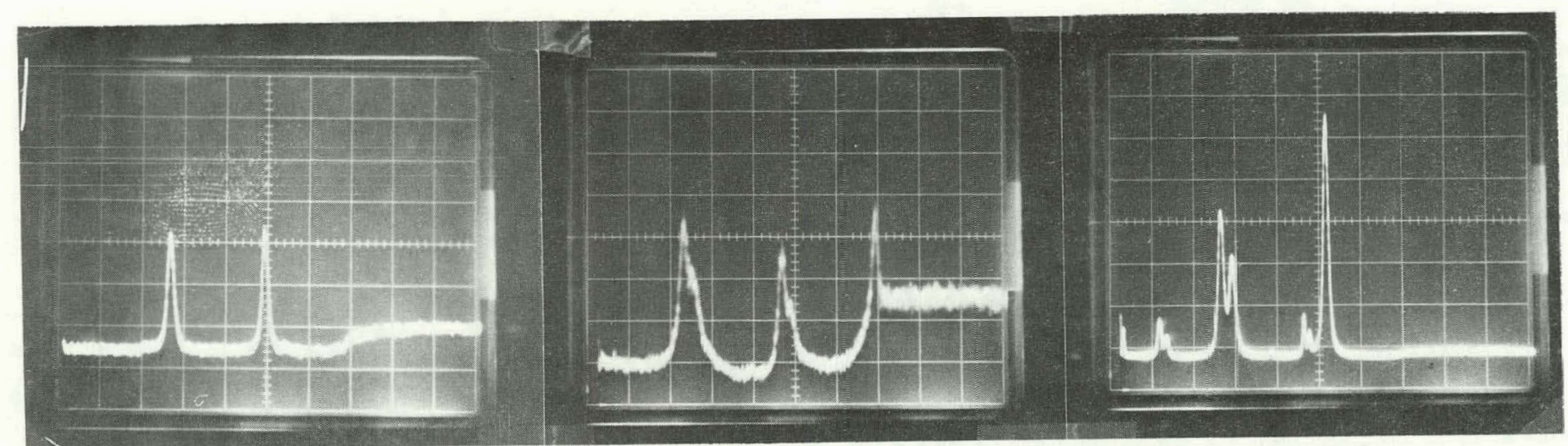

Figure 6. Signal traces for $10 \mathrm{ppm} K(\mathrm{a}), 30 \mathrm{ppm} R b(\mathrm{~b})$, and $10 \mathrm{ppm}$ In (c). Peaks and order of interference (m) from left to right are: (a) $\mathrm{K} 404.4 \mathrm{~nm}(\mathrm{~m}=70,71)$, (b) $\mathrm{Rb}$ doublet $420.2 \mathrm{~nm}(\mathrm{~m}=68,69)$ (c) In $410.4 \mathrm{~nm}(m=69), 451.1 \mathrm{~nm}(\mathrm{~m}=64), 680 \mathrm{~nm}$ molecular emission band. The small peaks shown in (c) are from $\mathrm{Na}$ in the deionized water 


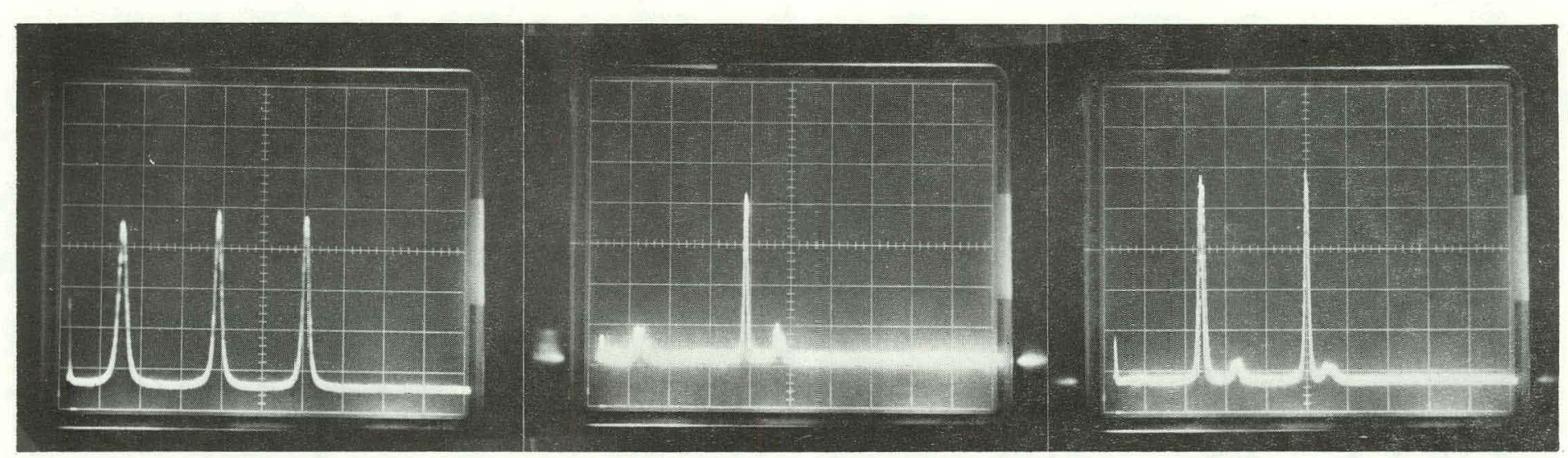

Figure 7. Signal traces for $3 \mathrm{ppm} \mathrm{Mn} \mathrm{(a),} 3 \mathrm{ppm} \mathrm{Li} \mathrm{(b),} \mathrm{and} 50 \mathrm{ppm} \mathrm{Sr} \mathrm{(c).}$ Peaks and order of interference $(n)$ from left to right are : (a) Mn $403.1 \mathrm{~nm}(\mathrm{~m}=70,71,72)$, (b) Li $670.8 \mathrm{~nm}(\mathrm{~m}=43)$, (c) $\mathrm{Sr}$ $460.7 \mathrm{~nm}(\mathrm{~m}=62,63)$. The small peaks in (b) and (c) are from $\mathrm{Na}$ in the deionized water 


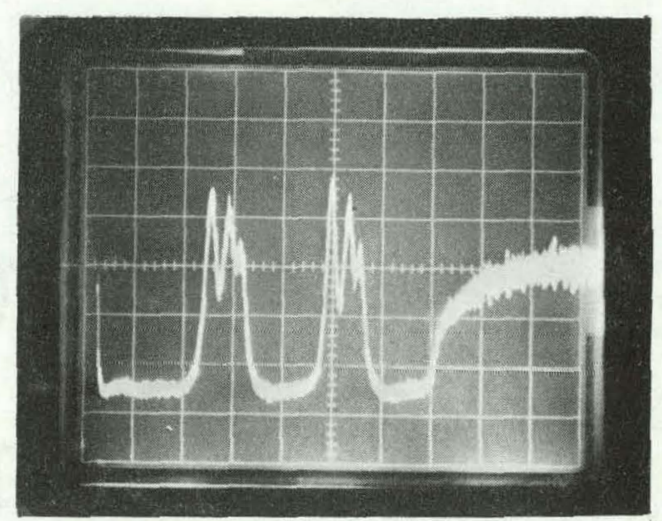

Figure 8. Signal trace of $50 \mathrm{ppm} \mathrm{Cr}$. Shown are two orders $(m=67,68)$ of the $425 \mathrm{~nm}$ triplet 
photographs may not be superimposed for the purpose of visualizing possible multielement combinations. In most cases, the distance traveled by the mirrors in a scan was enough to pass through two spacings that are consecutive integer multiples $(m, m+1$ in Equation 1$)$ of $\lambda / 2$ and, hence, most emission peaks appear twice (three times for Mn) in a single scan.

I'able $\perp$ presents the experimentally observed detection limits (defined for the point where $\mathrm{S} / \mathrm{N}=3$ ) for the ten elements. All stock solutions were prepared with reagent grade chloride salts in deionized water. Blanks run as checks showed the only element present at a detectable level in the deionized water was sodium at a concentration of .06 ppm (determined by standard addition). Thus the sodium detcotion limit reported in Table 1 represents a linear extrapolation from the observed $S / N$ for deionized water to the concentration at which $\mathrm{S} / \mathrm{N}=3$ would be expected. Observation of the emission in all cases was made at a height of $2.6 \mathrm{~cm}$ above the burner tip and no ionization buffers, releasing or protecting agents were added. Absolute detection limits were calculated by taking the product of the relative detection I limit, to tal time for analysis and the solution consumption rate. The parenthetical values 1 isted in Table 1 were determined from signal averaged spectra obtained with the computer 
Table 1. Detection limits

\begin{tabular}{|c|c|c|c|c|c|c|}
\hline \multirow{2}{*}{ Element } & \multirow{2}{*}{$\begin{array}{c}\begin{array}{c}\text { Analytical } \\
\text { Wavelength } \\
(\mathrm{nm})\end{array} \\
403.1\end{array}$} & \multicolumn{2}{|c|}{$\begin{array}{l}\text { Relative } \\
\text { Detection } \\
\text { Limit } \\
(\mu \mathrm{g} / \mathrm{mL})\end{array}$} & \multicolumn{2}{|c|}{$\begin{array}{l}\text { Absolute } \\
\text { Detection } \\
\text { Iimit } \\
\text { (ng) }\end{array}$} & \\
\hline & & .25 & $(.065)$ & .076 & $(2.7)$ & \\
\hline K & 404.4 & .75 & $(.024)$ & .22 & $(1.0)$ & \\
\hline $\mathrm{Rb}$ & 420.2 & 1.0 & $(.060)$ & .30 & $(2.5)$ & \\
\hline $\mathrm{Ca}$ & 422.7 & .10 & $(.019)$ & .031 & $(.82)$ & \\
\hline $\mathrm{Cr}$ & 425.4 & 1.2 & $(.22)$ & .36 & $(9.4)$ & \\
\hline In & 451.1 & .075 & $(.012)$ & .022 & $(.50)$ & \\
\hline Sr & 460.7 & .79 & $(.16)$ & .24 & $(6.9)$ & \\
\hline $\mathrm{Ba}$ & 553.6 & 3.0 & $(.55)$ & .90 & $(24)$. & \\
\hline $\mathrm{Na}$ & 589.0 & .009 & $(.307)$ & .0027 & $(.03)$ & \\
\hline $\mathrm{Li}$ & 670.8 & .15 & $(.1) 21)$ & .045 & $(.88)$ & \\
\hline
\end{tabular}


interface using a scan rate of $286 \mathrm{~Hz}$ and a data accumulation time of 3.5 sec $(1,000$ scans). Generally, the single scan detection limits given here are an order of magnitude poorer than those for single-element flame emission listed by Wineforaner (32). However, concentrations $1 / 10$ of those listed in Table 1 still produce a distinguishable signal when the averaging capability of the sample-hold circuit is utilized, thus confirming the possibilily uf detection at trace levels for all ten elements.

Elements which exhibited molecular emission show the poorest detection limits. If the background were totally of a random nature, an enhancement in the $S / N$ of $31.6(\sqrt{1000})$ would be expected' from the computer acquired data. However, only $\mathrm{Na}, \mathrm{Rb}$ and $\mathrm{K}$ show greater than a factor of 12 improvement in the detection limit after signal averaging indicating that detection is limited by background of a more continuous nature such as emission from molecular species (e.g. BaO in the case of $\mathrm{Ba}$ ) or the flame itself. Calibration plots of signal vs. concentration are shown in Figures 9 and 10. All of the elements display linearity over a minimum of three orders of magnitude in concentration with sodium providing a linear response from .06 to $1000 \mathrm{ppm}$. Either peak height or peak area may be used without significant difference however, the use of peak height is 


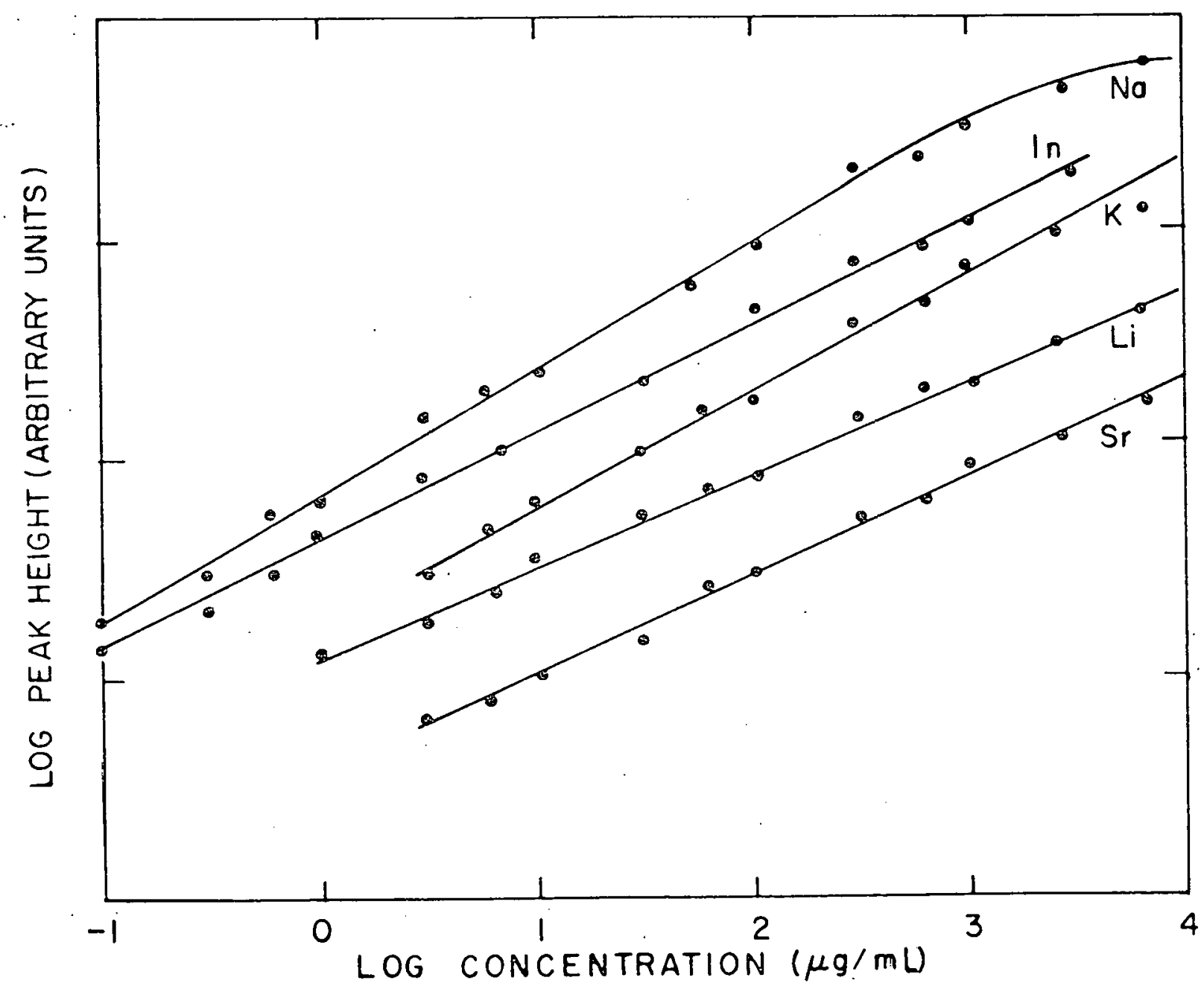

Figure 9. Calibration plots for aqueous solutions of $\mathrm{Na}$, In, K, Li and $\mathrm{Sr}$ 


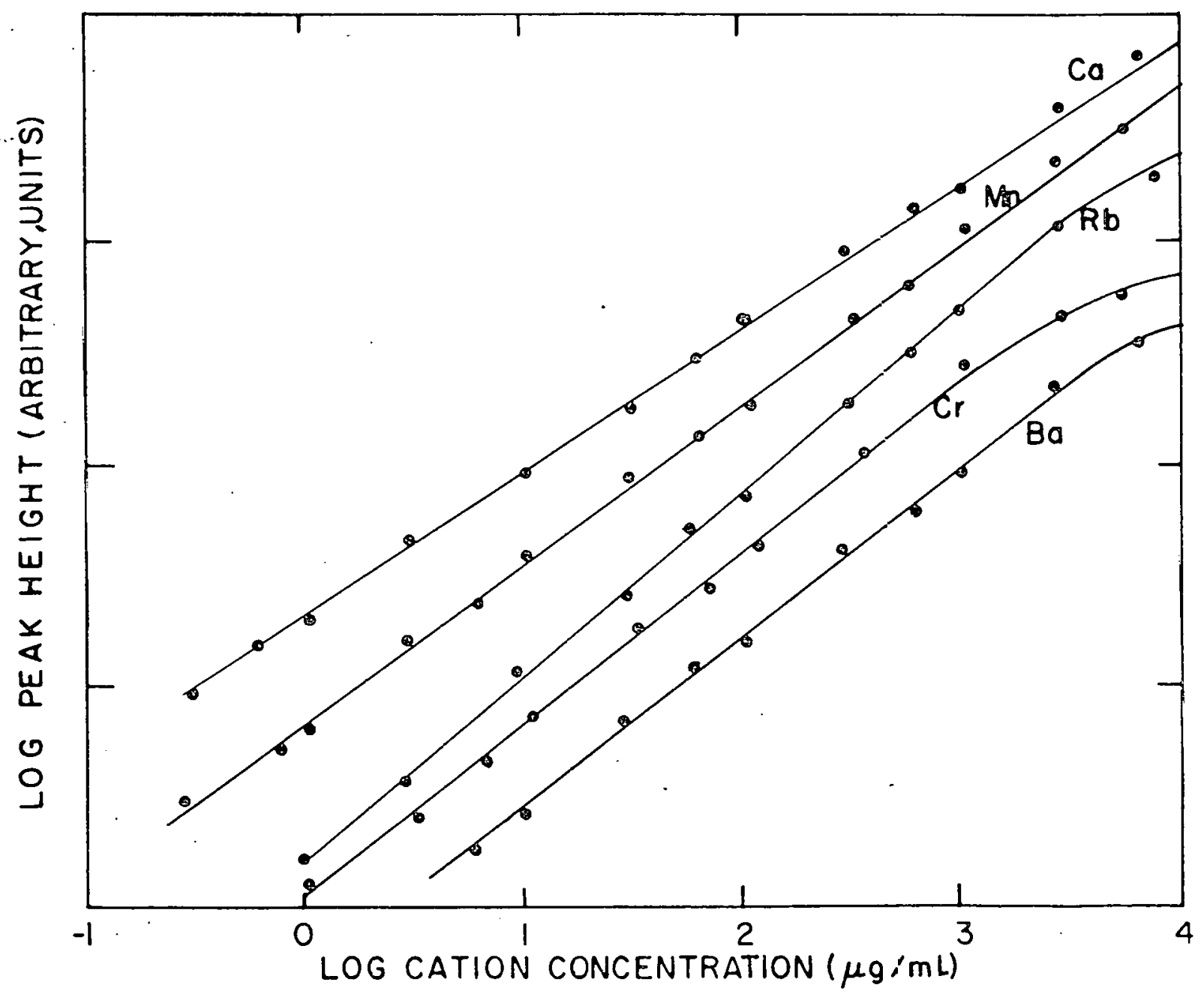

Figure 10. Calibration plots for aqueous solutions of $\mathrm{Ca}, \mathrm{Mn}, \mathrm{Rb}, \mathrm{Cr}$ and $\mathrm{Ba}$ 
preferred due to the lower probability of spectral interference. For $\mathrm{K}, \mathrm{Li}$ and $\mathrm{Rb}$, as the calibration plots are extended below the detection limit the curves acquire a steeper slope. This is most likely because these easily ionized atoms are known to exhibit high ionization effects at low concentrations (13). The relatively small flame $(10 \times 3 \mathrm{~cm})$ served as a sufficiently thin excitation source such that no self-absorption cffects were observed for any element at concentrations below $1000 \mathrm{ppm}$.

The interference effects of various anionic species present in matrices such as serum or urine were examined and are summarized in Table 2. Those elements not listed ( $R b$, In, Li) showed no appreciable effects with any of the five anions. In this study, a $100 \mathrm{ppm}$ solution of the cation prepared using the chloride salt is used as a reference and the values in Table 2 represent the percent reduction (-) or enhancement (+) of the emission signal in the presence of a tenfold excess of the interfering anion. All interferents were preparcd from the sodium salt except when the effects on sodium were tabulated, in which case the potassium salt was substituted. Pinta (107) provides an excellent discussion on the effects and mechanisms of chemical interferences encountered in flame spectrometry and the results noted in Table 2 generally compare favorably when conditions for analysis were similar. 
Table 2. Chemical interference effects

\begin{tabular}{|c|c|c|c|c|c|c|c|}
\hline \multirow[b]{2}{*}{ Interferent: } & \multicolumn{7}{|c|}{ Percent Change in Signal } \\
\hline & $\overline{\mathrm{Na}}$ & $\overline{\mathrm{K}}$ & $\mathrm{Sr}$ & $\mathrm{Ba}$ & $\mathrm{Ca}$ & $\mathrm{Cr}$ & $\overline{\mathrm{Mn}}$ \\
\hline $\mathrm{PO}_{4}$ & 0 & -.4 & -5 & -8 & -5 & +.1 & +.5 \\
\hline $\mathrm{SO}_{4}$ & 0 & +.5 & -3 & -4 & -.2 & 0 & +2 \\
\hline $\mathrm{CO}_{3}$ & -.2 & 0 & -.5 & -.3 & 0 & 0 & 0 \\
\hline $\mathrm{CH}_{3} \mathrm{CO}_{2}$ & 0 & +.1 & 0 & 0 & a & 0 & +1 \\
\hline $\mathrm{NO}_{3}$ & 0 & +1 & 0 & 0 & +1 & 0 & 0 \\
\hline
\end{tabular}


Of the three elements determined routinely in urine and serum ( $\mathrm{Na}, \mathrm{K}, \mathrm{Ca})$, only calcium exhibits any appreciable interference effects, however, when the $\mathrm{PO}_{4}$ to Ca ratio is lowered to levels similar to that present in these biological samples, only a 2 a suppression in Ca emission intensity was noted. Thus if the method of standard additions is used for analysis, problems due to matrix effects in these types of salnples will be minimal.

\section{B. Multielement Analysis}

This multielement analytical scheme makes use of the fact that most common elements have a limited number of atomic emission lines in the visible spectral range. Thus, when looking at this entire wavelength region, only the emission lines $m_{1} \lambda_{1}=2 d \cos \theta, m_{2} \lambda_{2}=2 d \cos \theta, \ldots$, will be transmitted simultaneously (see Equation 1 ) where $\lambda_{1}$ and $\lambda_{2}$ can be from the same or different elements. It is when this occurs that analysis using either $\lambda_{1}$ or $\lambda_{2}$ is prohibited but, since $\lambda_{1}$ and $\lambda_{2}$ are usually not whole numbers, the probability of any two lines being simultaneously transmitted at a givon $\mathrm{d}$ is very small. Although the finesse is what determines the ultimate number of resolvable lines in the scan (atomic linewidths are negligible here), the width of the single element peaks shown in Figures $5-8$ is determined not by the 
finesse but rather by the lack of collimation of the light incident upon the FP. So, with these widths, overlap of emission lines of differing wavelengths is possible. This is shown in Figure lla with a combination of $\mathrm{Ba}$ and $\mathrm{Na}$. The $553.6 \mathrm{~nm} \mathrm{Ba}$ and $589.0 \mathrm{~nm} \mathrm{Na}$ lines are transmitted essentially simultaneously at a mirror spacing of $14.9 \mu$. When this happens the peaks can generally be separated by using the interferometer in a different order (accomplished by changing the mirror spacing). This is illustrated in Figure 1lb where the mirror separation is now $20.5 \mu$ and the sodium and barium lines have been completely separated.

Using this approach, several synthetic multielement solutiono wore able to be analyzed without significant spectral overlap. These results are summarized in Table 3 which shows the range in inirror spacing that will result in the spectral separation of at least one order of one emission line for each element in a given mixture.

In order to assess the capabilities of the system for multielement analysis, five types of samples were chosen for study: tap water, urine, serum, orchard leaves and steel,. The element in these materials are present in a variety of matrices and therefore provide a means of examining the scanning FP optical system for rapid multielement analysis of real samples. In all cases detection was accomplished using a $25 \mathrm{msec}$ scan rate with peak intensities being repetitively 
(a)

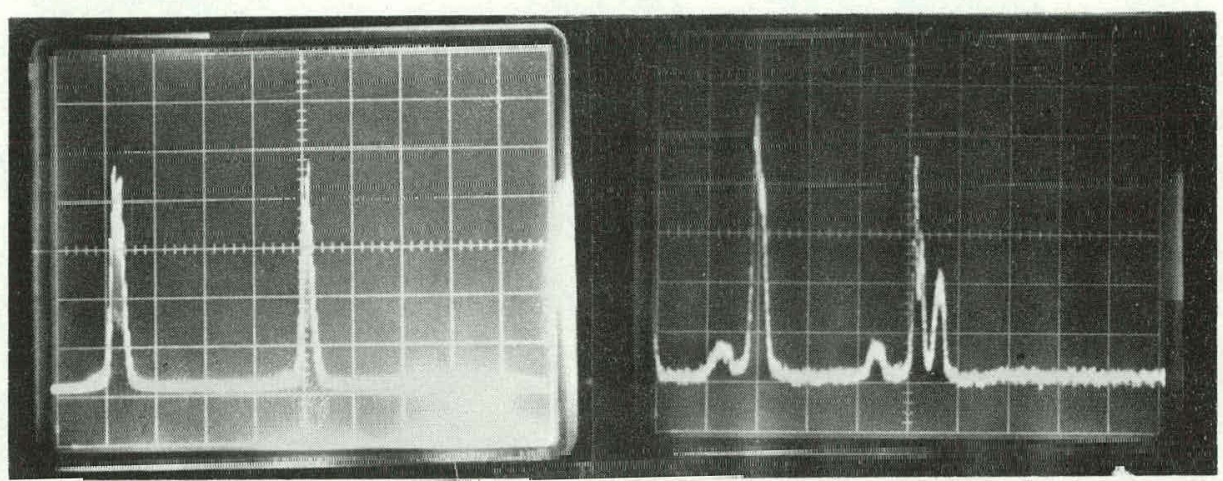

Figure 11. Resolving a mixture of $\mathrm{Na}$ and $\mathrm{Ba} .10 \mathrm{ppm} \mathrm{Ba}$ and $1 \mathrm{ppm} \mathrm{Na}$ (a) at a mirror spacing of $14.9 \mu$. In b, $10 \mathrm{ppm} \mathrm{Ba}, 1 \mathrm{ppm} \mathrm{Na}$ and $10 \mathrm{ppm} \mathrm{Li}$ are shown at a spacing of $20.5 \mu$. Peaks in $\mathrm{b}$ from left to right are: $\mathrm{Ba}, \mathrm{Na} / \mathrm{Li}$ overlap, $\mathrm{Ba}, \mathrm{Na}$ and $\mathrm{Li}$. Signal was attenuated using a Corning CS-1-60 filter 
Table 3. FP mirror spacings required for resolution of certain multielement combinations

Elements present

Mirror spacing $(\mu)$

$\begin{array}{ll}\mathrm{Ba}, \mathrm{K}, \mathrm{Na}, \mathrm{Li} & 20.4-21.1 \\ \mathrm{Na}, \mathrm{Li}, \mathrm{K}, \mathrm{Ca} & 14.0-17.0 \\ \mathrm{~K}, \mathrm{Mn}, \mathrm{Rb}, \mathrm{Ca} & 14.0-18.0 \\ \mathrm{Cr}, \mathrm{Mn}, \mathrm{Sr} & 14.0-18.0 \\ \mathrm{Cr}, \mathrm{Mn}, \mathrm{In} & 14.0-20.0 \\ \mathrm{Cr}, \mathrm{Mn}, \mathrm{Li} & 14.0-18.0\end{array}$

sampled for a $45 \mathrm{sec}$ time period so as to utilize the signal averaging capability of the sample-hold circuit. To compensate for fluctuations in excitation conditions over a longer period of time, peak heights were normalized to an internal standard (usually Li) when possible. Figure 12 demonstrates the effect of using a $\mathrm{Li}$ internal standard for the determination of $\mathrm{Ca}$ in tap water. The fluctuations in signal intensity for the Ca $422.7 \mathrm{~nm}$ emission line caused by aspiration rate variations, improper sample manipulations and fuel-to-oxidant ratio shifts is minimized by normalizing the intensity of the Ca line to that of the Li. The advantages for the use of internal standards in flame spectrometry have been discussed thoroughly by Barnett, Fassel and Kniseley 


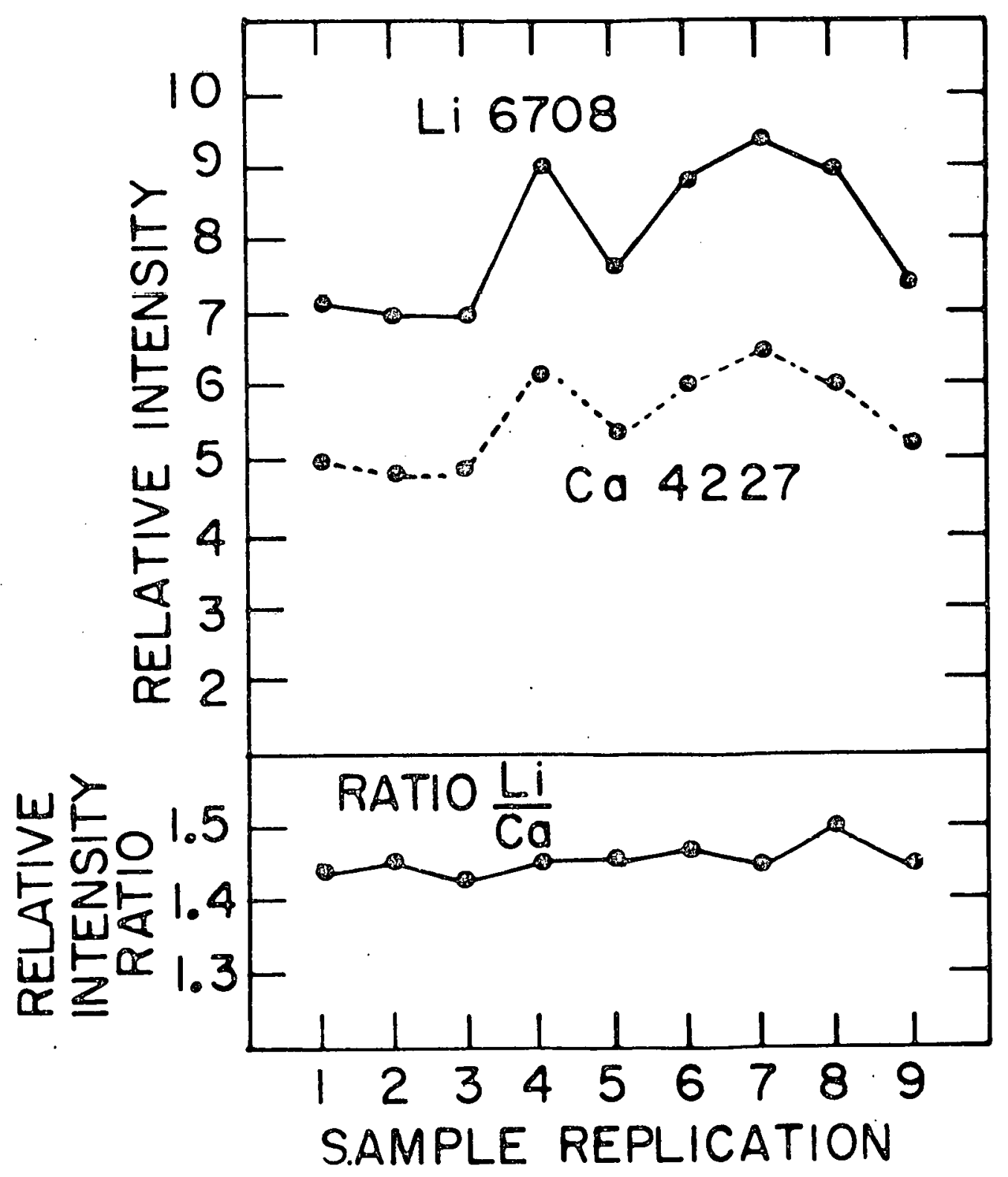

Figure 12. Use of a lithium internal standard for the determination of calcium in tap water 
(108). Analytical determinations for all samples were made by the addition of aqueous standard solutions with the usual graphical evaluation of the results (109).

\section{Tap water}

A signal trace of $\mathrm{Ca}$ and $\mathrm{Na}$ in laboratory tap water is shown in Figure 13. Due to the extreme sensitivity to sodium, the emission radiation was attenuated with a Corning CS-1-64 blue filter. This filter reduces the Na intensity by about $90 \%$ while decreasing the Ca emission intensity by only $10 \%$. Table 4 shows the results obtained on a laboratory tap water sample. For comparison, the $\mathrm{Na}$ analysis was also done using a Beckmann Model DU quartz-prism spectrometer and the Ca by EDTA titration (10.9). The mean $(\bar{X})$, standard deviation (S) and relative standard deviation $(S / \bar{X})$ are given in each instance.

All peak heights were normalized to a lithium internal standard. Flame emission methods of analysis usually provide precision an order of magnitude poorer than that obtainable with titrimetric procedures and the FP is no exception to this generalization. However, when compared to the singleelement DU technique, the FP provides as good or better precision in all cases and in addition, consumes a smaller amount of analyte and requires less analysis time than either of the other two methods. 


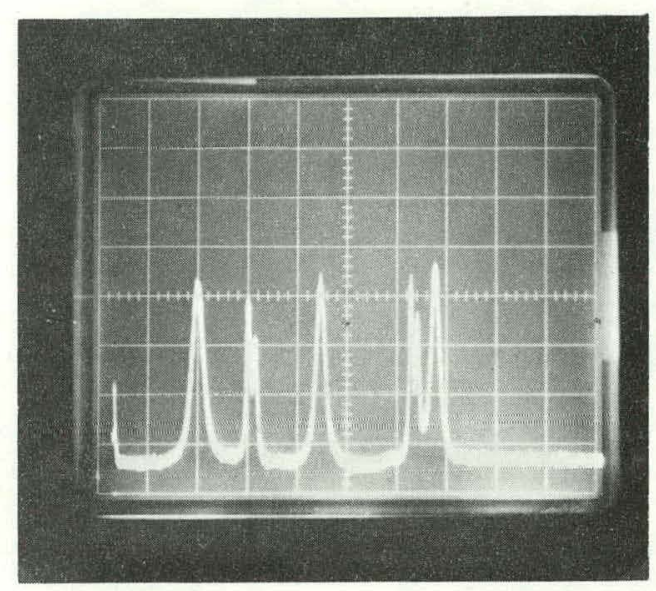

Figure 13. Direct aspiration of laboratory tap water containing $100 \mathrm{ppm} \mathrm{Ca}$ and $33 \mathrm{ppm} \mathrm{Na}$. Peaks, from lett to right are: $\mathrm{Ca}, \mathrm{Na}, \mathrm{Ca}, \mathrm{Na}$ and $\mathrm{Ca}$. Signal is attenuated using a Corning CS-1-64 filter 
Table 4. Analysis of tap water

\begin{tabular}{ccccc}
\hline Trial & \multicolumn{2}{c}{$\mathrm{Na}(\mathrm{mg} / \mathrm{L})$} & \multicolumn{2}{c}{$\mathrm{Ca}(\mathrm{mg} / \mathrm{L})$} \\
\hline $\mathrm{FP}$ & 35 & $\mathrm{DU}$ & 100 & 95.0 \\
2 & 30 & 37 & 97 & 95.4 \\
3 & 32 & 35 & 99 & 95.5 \\
4 & 35 & 38 & 104 & 95.9 \\
5 & 34 & 37 & 101 & 95.8 \\
$\overline{\mathrm{X}}(\mathrm{mg} / \mathrm{L})$ & 32 & 37.4 & 100.2 & 95.52 \\
$\mathrm{~S}(\mathrm{mg} / \mathrm{L})$ & 33.6 & 1.8 & 2.6 & .35 \\
$\mathrm{~S} / \overline{\mathrm{X}}(\mathrm{\circ})$ & 1.5 & 4.8 & 2.6 & .37 \\
& 4.4 & & & \\
\hline
\end{tabular}




\section{Urine and serum}

The problems of the analytical biochemist differ from the usual concerns of other analysts in two special ways. On the one hand the amount of initial sample is usually limited, in the case of serum to a few $\mathrm{mL}$. On the other hand, multielement information is often required from these small samples. Determining the sodium, potassium and calcium levels in biological fluids is considered a very routine and necessary procedure in clinical laboratories. Normal. serum concentrations of sodium, potassium and calcium are $3.1-5.6, .14-.19$ and $.75-1.5 \mathrm{mg} / \mathrm{ml}$, respectively. Concentrations in urine are greater still.

Twenty-four hour urine samples were collected from 5 subjects and refrigerated until analysis. Five vials of dehydrated human blood serum were obtained from the Dade Division of the American Hospital Supply Company and reconstituted in $5 \mathrm{ml}$ of deionized water. A five $\mathrm{mL}$ portion of each specimen was prepared for analysis by adding $25 \mathrm{mg}$ of Ii and $25 \mathrm{ml}$ of $2 \%$ sterox (a surfactant solution that prevents clogging of the nebulizer) and diluting to $250 \mathrm{~mL}$ with deionized water. Signal. traces for $\mathrm{Na}, \mathrm{K}$ and $\mathrm{Ca}$ in urine and serum are shown in Figures $14 \mathrm{a}$ and $\mathrm{b}$. A Corning CS-1-60 didymium filter was used to attenuate the sodium emission intensity and quantitation was accomplished via standard addition analysis. Results for several specimens are 
(a)

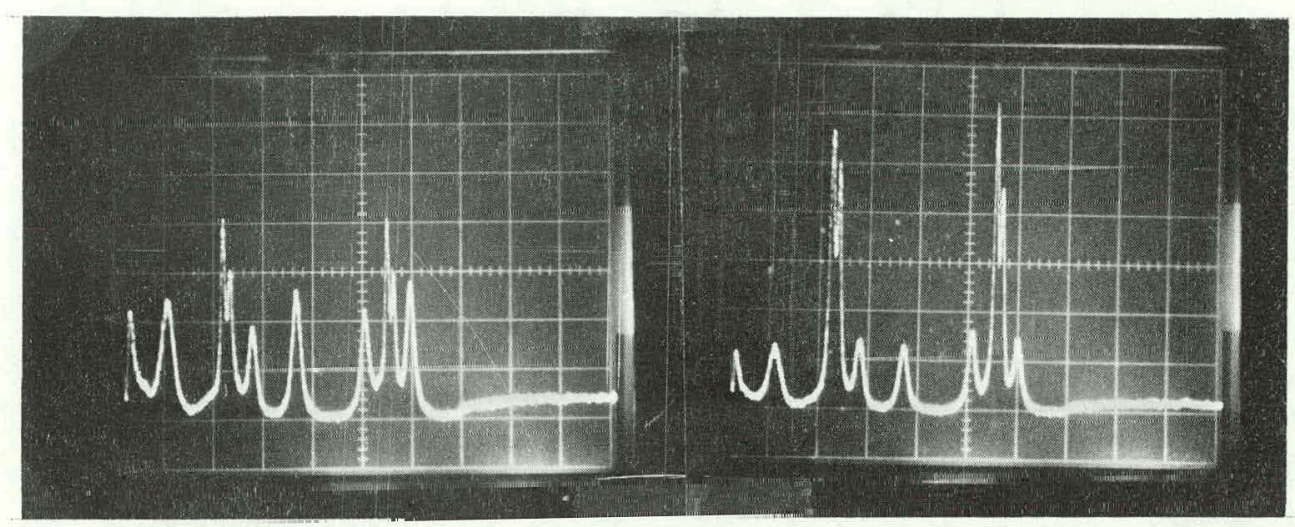

Figure 14. Signal traces for $160 \mathrm{ppm} \mathrm{Na,} 70 \mathrm{ppm} \mathrm{K}$ and 60 ppm $\mathrm{Ca}$ in urine (a) and $75 \mathrm{ppm} \mathrm{Na}, 15.8 \mathrm{ppm} \mathrm{K}$ and $12 \mathrm{ppm} \mathrm{Ca}$ in serum (b). Peaks in both cases are (from left to right): $\mathrm{Ca}, \mathrm{Na}, \mathrm{K}, \mathrm{Ca}, \mathrm{K}, \mathrm{Na}$ and Ca. A Corning CS-1-60 filter was used to the attenuate $\mathrm{Na}$ intensity 
presented in Table 5. The values listed for the urine represent $\mathrm{mEq}$ present in a 24-hour sample and are compared to results obtained using a Beckmann Model DU quartz spectrometer. Analytical determinations for the serum samples are presented in the units most often given in the literature and are compared to the assay values supplied by the manufacturer (110).

Generally, accuracy within $\%$ of the actual value was observed for the serum samples while results obtained on the urine specimens indicate close agreement with those acquired using the Beckmann DU. Precision for both types of samples was usually less than $3 \%$ which is as good or better than the precision reported with commercial clinical multielement flame photometers (111).

\section{Orchard leaves}

Historically, flame emission photometry has been the method of choice for the determination of $\mathrm{K}, \mathrm{Ca}$ and $\mathrm{Na}$ in agricultural materials. To evaluate the potential of such an application to the FP system, NBS SRM 1571 orchard leaves were analyzed for the three above mentioned elements. One gram samples of dry orchard leaves were dissolved in $50 \mathrm{~mL}$ of a $3: 2 \mathrm{HNO}_{3} / \mathrm{HClO}_{4}$ solution and then diluted to $500 \mathrm{~mL}$. A biank containing the $\mathrm{HNO}_{3} / \mathrm{HClO}_{4}$ and deionized water was also prepared. Analysis for $\mathrm{Na}, \mathrm{K}$ and $\mathrm{Ca}$ by standard addition 
Table 5. Analysis of urine and serum

\begin{tabular}{c} 
Patient \\
\hline
\end{tabular}

A. Urine

$\begin{array}{rrrrrrr}1 & 190 & 187 & 210 & 202 & 120 & 110 \\ 2 & 110 & 115 & 120 & 115 & 90 . & 92 \\ 3 & 170 & 175 & 130 & 120 & 50 & 55 \\ 4 & 180 & 184 & 180 & 175 & 100 & 96 \\ 5 & 120 & 130 & 95 & 90 & 130 & 127\end{array}$

\begin{tabular}{|c|c|c|c|c|c|c|}
\hline \multirow[b]{2}{*}{ erum } & $\mathrm{Na}$ & $(\mathrm{mEq} / \mathrm{L})$ & $\mathrm{Ca}$ & $g(d L)$ & \multicolumn{2}{|c|}{$\mathrm{K}(\mathrm{mEq} / \mathrm{L})$} \\
\hline & $\overline{\mathrm{FP}}$ & A.SSAY & $\overline{\mathrm{FP}}$ & $\triangle \widehat{A S A Y}$ & $\overrightarrow{F P}$ & ASSAY \\
\hline 1 & 140 & 149 & 10 & 9.9 & 2.5 & 2.7 \\
\hline 2 & 143 & 149 & 8.2 & 8.5 & 5.2 & 4.9 \\
\hline 3 & 130 & 127 & 11 & 11.5 & 3.1 & 3.6 \\
\hline 4 & 105 & 112 & 8.5 & 8.1 & 3.7 & 4.0 \\
\hline 5 & 138 & 140 & 9.2 & 9.6 & 4.2 & 4.5 \\
\hline
\end{tabular}

was performed using a Corning cs-3-77 yellow filter to reduce the intensity of the $\mathrm{Ca}$ and $\mathrm{K}$ emission which are prescnt at much higher levels than the sodium. Although it is a trace component in the orchard leaves $(14 \mu \mathrm{g} / \mathrm{g})$, no signal from $\mathrm{Li}$ was distinguishable in the solution prepared and thus $\mathrm{Li}$ was added as an internal standard. Figure 15 shows a signal trace of the orchard leaves solution and 


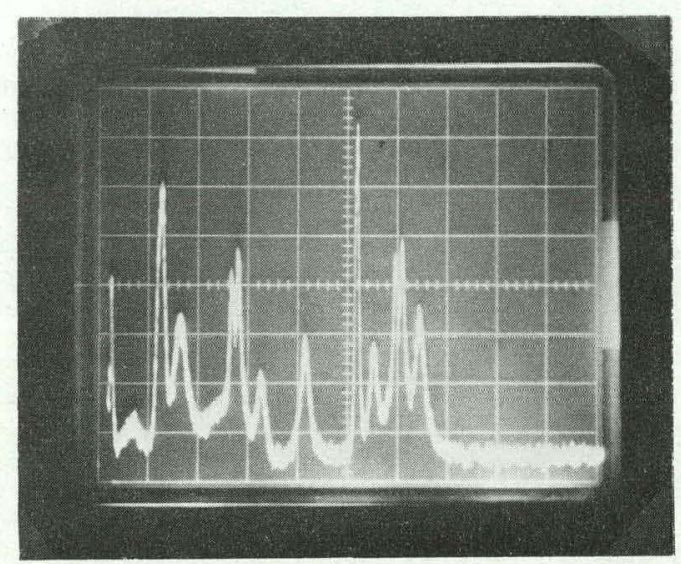

Figure 15. Signal trace for $42 \mathrm{ppm} \mathrm{Ca}, 30 \mathrm{ppm} \mathrm{K}$ and $.17 \mathrm{ppm}$ $\mathrm{Na}$ in NBS orchard leaves with a $50 \mathrm{ppm} \mathrm{Li}$ internal standard. From left to right, peaks are: Li, Ca, $\mathrm{Na}, \mathrm{K}, \mathrm{Ca}, \mathrm{Li}, \mathrm{K}, \mathrm{Na}$ and $\mathrm{Ca}$ 
Table 6 the statistical results obtained for several trials. Precision (1.6-3.9\%), and accuracy (1.7-6.2\%), are generally better than conventional single-element methods for samples of similar origin (34).

4. Steel

The determination of chromium and manganese in steels and cast iron has been of interest for many years. NBS SRM 163 is a powdered low alloy chromium steel which contains both $\mathrm{Cr}$ and $\mathrm{Mn}$ at slightly less than a $1 \%$ concentration.

Table 6. Analysis of NBS SRM 1571 orchard leaves

\begin{tabular}{cccc}
\hline Trial & $\mathrm{Ca}(\mathrm{mg} / \mathrm{g})$ & $\mathrm{K}(\mathrm{mg} / \mathrm{g})$ & $\mathrm{Na}(\mu \mathrm{g} / \mathrm{g})$ \\
\hline 1 & 19.5 & 14.5 & 80.6 \\
2 & 20.5 & 13.7 & 82.4 \\
3 & 19.0 & 15.1 & 79.8 \\
4 & 19.9 & 14.2 & 81.3 \\
5 & 19.2 & 13.8 & 78.9 \\
$\overline{\mathrm{X}}$ & 19.6 & 14.3 & 80.6 \\
S & .60 & .57 & 1.3 \\
S/X (\%) & 3.0 & 3.9 & 1.6 \\
Certified Value & 20.9 & 14.7 & 82.0 \\
\hline
\end{tabular}


One gram portions of the alloy were dissolved in. $50 \mathrm{~mL}$ of concentrated nitric acid, $60 \mathrm{mg}$ of $\mathrm{Sr}$ were added and the solution was diluted to $500 \mathrm{~mL}$ with deionized water. No silica or graphite was present after dissolution and thus no filtration was necessary prior to analysis. A corning CS-1-60 didymium filter was used to essentially eliminate the emission intensity from the Na present in the blank. A signal trace for the steel solution is shown in Figure 16. Chromium and manganese peak heights were normalized to the Sr emission intensity and quantitatively determined by standard addition. Results from five analyses are summarized in Table 7 .

Table 7. Analysis of NBS SRM 163 low alloy steel

\begin{tabular}{lll}
\hline Trial & Mn $(\mathrm{mg} / \mathrm{g})$ & $\operatorname{Cr}(\mathrm{mg} / \mathrm{g})$ \\
\hline 1 & 8.4 & 9.2 \\
2 & 8.5 & 9.0 \\
3 & 8.8 & 9.2 \\
4 & 8.4 & 9.4 \\
5 & 8.3 & 9.5 \\
$\bar{X}(\mathrm{mg} / \mathrm{g})$ & 8.48 & 9.36 \\
$\mathrm{~S}(\mathrm{mg} / \mathrm{g})$ & .19 & .20 \\
S/X (o) & 2.2 & 2.0 \\
Certified Value & 8.97 & 9.82 \\
\hline
\end{tabular}




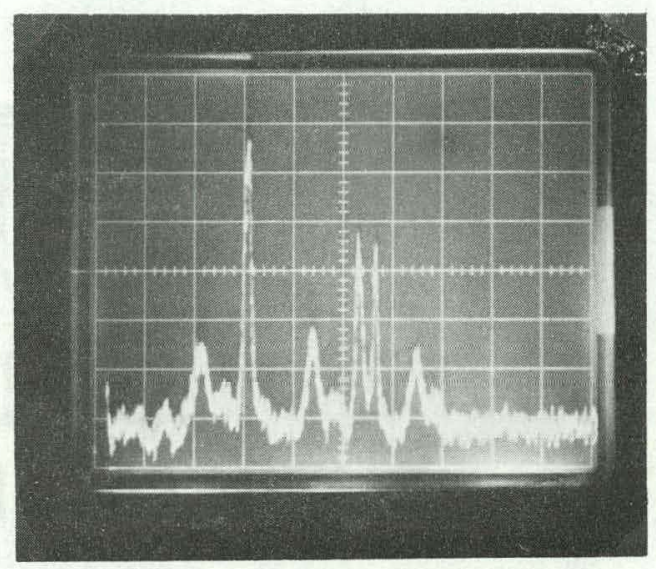

Figure 16. Signal trace for $18 \mathrm{ppm} \mathrm{Mn}$ and $20 \mathrm{ppm} \mathrm{Cr}$ in NBS low alloy steel. A $10 \mathrm{ppm}$ Sr internal standard is used. Peaks from left to right are: $\mathrm{Cr}$, $\mathrm{Mn} / \mathrm{Sr}$ overlap, $\mathrm{Cr}, \mathrm{Mn}, \mathrm{Sr}$ and $\mathrm{Cr}$ 
On similar NBS alloys, Fassel et al. (112) reported a 1\% relative standard deviation with an accuracy of $8 \%$ using single-element flame emission analysis. This work demonstrated a precision of $2 \%$ with only a 5\% deviation from the certified value. 


\section{SUMMARY}

In this study, a system for rapid multielement flame emission analysis based upon a scanning FP interferometer with a single detector was improved from the original design by Pruiksma etal. (1) and shown to be applicable to the analysis of a variety of samples. Coupled to a simple multichannel peak height sampling system, the analytical scheme shows excellent detection limits and linearity of response for ten elements having emission lines in the visible spectral region with precision and accuracy comparable and in many instances superior to conventional multielement techniques.

other advantages of the system include the small volumes of analyte required, the ease of changing to different groups of elements, the small amount of analyte required, and the low cost of the total analytical arrangement (less than $\$ 3,500$ ).

The method is not without its disadvantages, however. With the current design, only four or possibly five elements may be determined simultaneously and thus samples which contain more than five elements at detectable levels are not easily analyzed. The number of elements may be increased by careful collimation of the light incident upon the FP but this in turn decreases the spectral throughput of the system. Unlike a monochromator, the interferometer requires finetuning at weekly intervals and its alignment is critical to 
obtaining accurate, reproducible results. Molecular emission bands can not be used for analysis, therefore requiring that the flame be adjusted to ensure the greatest degree of dissociation of molecular species.

It is not expected that the FP interferometer will replace the usual dispersive emission spectrometer for most applications. However, this work has shown that for analysis of samples with a relatively simple composition, the FP offers distinct advantages not present in other multielement optical systems. 
VI. SUGGESTIONS FOR FUTURE STUDY

The most important area for improvement of the technique still lies in optimization of the optical system for increased resolution while maintaining acceptable limits of detection. There are two approaches which can be taken in an effort to solve this problem. The first would utilize the same optical concept as was described here but a longer focal length lens would be substituted for the $200 \mathrm{~mm}$ focal length lens (L2). shown in Figure 1. This would allow the angle: $\theta$ in Equation 1 to become smaller and thus serve to narrow the transmitted peak width.

The second approach would be to utilize a collimation system similar to that described by Pruiksma etal. (1) coupled with better collection efficiency of the radiation emanating from the source. The use of an electric spark would certainly be a great improvement. The spark localizes the emission such that collection efficiency as well as collimation can be much improved. The spark would also take advantage of the short duration of the detection scan of the FP.

Obviously, as the resolution of the system is improved, samples of more complicated composition may be analyzed including coal, petroleum, geological and biological materials. Also, since a greater number of elements have their most sensitive emission lines in the ultraviolet (UV) region of the 
spectrum, replacement of the present FP mirrors with a set of UV-transmitting mirrors would allow application of the system to a different group of elements including most of the transition metals. The mirrors are easily changed and this would allow the technique to be applied to an even wider variety of analytical problems. 


\section{BIBLIOGRAPHY}

1. R. Pruiksma, J. Ziemer and E. Yeung, Anal. Chem. 48 , $667(1976)$.

2. A. Ahearn, Ed., Trace Analysis by Mass Spectrometry (Academic Press, New York, 1972).

3. R. Boulton and G. Ewan, Anal. Chem. 49, 1297 (1977).

4. R. Nadkarni and G. Morrison, Anal. Chem. 45, 1957 (1973).

5. P. Clark, G. Neal and R. Allen, Anal. Chem. 47, 650 (1975).

6. M. Agarwal, R. Bennett, I. Stump and J. D'Auria, Anal. Chem. 47, 924 (1975).

7. R. Giauque, R. Garrett and L. Goda, Anal. Chem. 49, 62 (1977).

8. A. Mittler, B. Barnes, R. Litman, F. Holton and E, Barry, Anal. Chem, 49, 43? (1977).

9. A. Bond and J. Carterford, Anal. Chem. 44, 732 (1972).

10. A. Borello and G. Guidotti, Anal. Chem. 43, 607 (1971).

11. W. G. Richards and P. R. Scott, Structure and spectra of Atoms (Wiley, New York, 1976).

12. B. Straughan and S. Walker; Spectroscopy (Chapman and Hall, London, 1976) Volume I, Chapter 1 .

13. J. Winefordner, Ed., Spectrochemical Methods of Analysis (Wiley-Interscience, New York, 1971) Chapter 2.

14: E. Condon and G. Shortley, The Theory of Atomic Spectra (Cambridge University Press, London, 1970).

15. G. Herzberg, Atomic Spectra and Atomic Structure (Dover, New York, 1944). 
16. R. Mavrodineanu and H. Boiteux, Flame Spectroscopy (Wiley, New York, 1965).

17. J. Winefordner, W. McGee, J. Mansfield, M. Parsons and K. Zacha, Anal. Chim. Acta 36,25 (1966).

18. E. L. Grove, Ed., Analytical Emission Spectroscopy (Decker, New York, 1971), Chapter 1, Part I, Part II.

19. P. Noumans, Theory of: Spectrochemical Excitation (Adam Hilger, London, I966).

20. M. Slavin, Emission Spectrochemical Analysis (WileyInterscjence, New York, 1971), Chpater 7.

21. G. Morrison, Trace Analysis-Physical Methods (Wiley-Interscience, New York, 1965).

22. C..Keirs and T. Vickers, Appl. Spectrosc. 31, 273 (1977).

23. V. Fassel and R. Kniseley, Anal. Chem. 46, 110A (1974).

24. Ibid., 1155A.

25. P. Boumans and F. de Boer, spectrochim. Acta 27B, 391 (1972).

26. R. Scott, V. Fassel, R. Kniseley and D. Nixon, Anal. Chem. 46, 75 (1974).

27. G. Kirchhoff and R. Bunsen, Ann. Physik (Poggendorf's Ann, ) 110, 161 (1860).

28. M. Janssen, Compt. Rend. 71, 626 (1870).

29. R. Barnes, D. Richardson, J. Berry and R. Hood, Ind. Eng. Chem. (Anal. Ed.) .17, 605 (1945).

30. F. Burriel-Martí and J. Ramírez-Muñoz, Fotometría de Ilama (Monografías de Ciencia Moderna, Consejo Superior de Investigaciones Científicos, Madrid, 1955).

31. W. Schrenk, Analytical Atomic Spectroscopy, (Plenum Press, New York, 1975). 
32. J. Winefordner, Trace Analysis, Spectroscopic Methods for Elements (Wiley, New York, 1976), Chapters 6, 11.

33. J. Dean and T. Rains, Flame Emission and Atomic Absorption Spectrometry (Marcel Dekker, New York, 1969), Volume 1 .

34. R. Mavrodineanu, Ed., Analytical Flame Spectroscopy (Springer-Verlag, New York, 1970).

35. R. Winge, V. Fassel and R. Kniseley, Appl. Spectrosc. 25, 636 (1971).

36. W. Harrison and P. Juliano, Anal. Chem. 43, 248 (1971).

37. L. de Galan and J. Wincfordner, J. Quant. Spectry. Radiative Transfer 2,703 (1967).

38. E. Pickett and S. Koirtyohann, Anal. Chem. 41 (14), 28 A (1969).

39. R. Mavrodineanu, Bibliography. on Flame Spectroscopy Analytical Applications 1800-1966 (Natl. Bur. Std. (U.S.) Misc. Publ.281, Washington, D.C., 1967).

40. H. Shroeder and A. Nason, Clin. Chem. 17, 461 (1971).

41. E. Underwood, Trace Elements in Human and Animal Nutrition, 3rd Ed. (Academic Press, New York, 1971).

42. W. Mertz and W. Cornatzer, Newer Trace Elements in Nutrition (Marcel Dekker, New York, 1.97I).

43. E. Berman, Appl. Spectrosc. 29, 1 (1975).

44. W. White, M. Erickson and S. Stevens, Chemistry for the Clinical Laboratory, 4 th Ed. (Mosley, St. Louis,. 1976), Chapter 5.

45. R. Henry, D. Cannon and J. Winkelman, Clinical Chemistry Principles and Technics, 2nd Ed. (Harper and Row, New York, 1974 $\longdiv { \text { , Chapter } 1 9 }$.

46. R. Dagnall, G. Kirkbright, T. West, and R. Wood, Anal. Chem. 43, 1765 (1971). 
47. P. Hannaker and T. Hughes, Anal. Chem. 49, 1485 (1977).

48. R. Ruch, H. Gluskoter and N. Shimp, Occurrence and Distribution of Potential Volatile Trace Elements in Coal, Illinois state Geological Survey, August, 1974.

49. O. Milner, Analysis of Petroleum for Trace Elements (Pergamon press, New York, 1963).

50. V. Fassel, R. Slack and R. Kniseley, Anal. Chem. 43, 186 (1971).

51. J. Winefordner, J. Fitzgerald and N. Omenetto, $\Lambda$ ppl. spectrose. 29, 369 (1975).

52. A. Harris and J. Jackson, J. Phys. E: Sci. Instrum. 3. 374 (1970).

53. H. Kruegle and S. Dolin, Appl. Opt. 8,2107 (1969).

54. D. Dieke and H. Crosswhite, J. Opt. Soc. Amer. 35 , 471 (1945).

55. D. Dye and I. Fel.Aman, Rcv. 3ui. Instrum. 37, 154 (1966).

56. R. Brehm and V. Fassel, J. Opt. Soc. Amer. 43, 886 (1953).

57. R. Santini, M. Milano and H. Pardue, Anal. Chem. 45, 915A (1973).

58. G. Pimentel, Appl. Opt. 7, 2155 (1968).

59. J. Dawson, D. Ellis and R. Milner, Spectrochim. Acta 23B, 695 (1968).

60. E. Cordos and H. Malmstadt, Anal. Chem. 45, 425 (1973).

61. D. Johnson, F. Plankey and J. Winefordner, Can. J. Spectrosc. 19, 151 (1974).

62. D. Mitchell and A. Johansson, Spectrochim. Acta 25B, $175(1970)$.

63. D. Mitchell and A. Johansson, Spectrochim. Acta 26B, 677 (1971). 
64. R. Elser and J. Winefordner, Appl. Spectrosc. 25, 345 (1971).

65. H. Hurter and V. Driffield, J. Soc. Chem. Ind. 9 , 455 (1890).

66. W. McGucken, Nineteenth-Century Spectroscopy (Johns Hopkins Press, Baltimore, 1970).

67. H. Dingle, A.Hundrcd Years of Spectroscopy (Blackwell, Oxford, $195 \overline{1)}$.

68. B. Vallee and M. Margoshes, Anal. Chem. 28, 1975 (1956).

69. R. Mavrodineanu and R. Hughes, Appl. Opt. 7, 1281 (1968).

70. L. Butler and A. Strásheim, Spectrochim. Acta 21, 1207 (1965).

71. A. Strasheim and H. Human, Spectrochim. Acta 23B, 265 (1968).

72. V. Fassel and R. Kniseley, Anal. Chem. 46, 1155A (1974)

73. A. Johansson and L. Nilsson, Spectrochim. Acta 31B, $419(1976)$.

74. V. Maslov, J. Appl. Spectrosc., USSR 2, 131 (1965).

75. W. Snelleman, T. Rains, K. Yee, H. Cook and O. Menis, Anal. Chem. 42 394 (1970).

76. U. Haisch, K. T,aqua and and $\mathrm{W}$. Hagenah, Spectrochim. Acta 26B, 651 (1971).

77. M. Margoshes, Spectrochim. Acta 25B, 113 (1970).

78. R. Benn, W. Foote and C. Chase, J. Opt. Soc. Amer. 39,529 (1949).

79. D. Mitchell, K. Jackson and K. Aldous, Anal. Chem. 45, 1215A (1973).

80. K. Busch, N. Howell, G. Morrison, Anal. Chem. 46 , 575 (1974). 
81. Y. Talmi, Anal. Chem. 47, 658A (1975).

82. G. Horlick, Appl. Spectrosc. $\underline{30}, 113$ (1976).

83. P. Boumans and G. Brouwer, Spectrochim. Acta 27B, 247 (1972).

84. J. Caunes and W. Kosonocky, RCA Rev. 33, 607 (1972).

85. Development Note, Opt. Spectra (January 1974).

86. C. Fabry and A. Perot, Annls. Chim. Phys. Ser 812 , 459 (1897).

87. F. Jacquinot, J. Opt. Soc. Am. 44, 761 (1954).

88. K. Mielenz, R. Stepehsn, K. Gillilland and K. Nefflen. J. Opt. Soc. Am. 56, 156 (1966).

89. D. Jackson and H. Kuhn, Proc. Roy. Soc. A 149, 372 (1936).

90. D. Bradley, Proc. Roy. Soc. A 262, 529 (1961).

91. P. Brannon and F. Bacon, Appl. Opt. 12, 142 (1973).

92. G. Kirkbright, o. Troccoli and $\mathrm{S}$. Vetter, spectrochim. Acta 28B, 1 (1973).

93. C. Veillon and P. Merchant, Jr., Appl. Spectrosc. 27 , 361 (1973).

94. S. Tolansky, Introduction to Interferometry (Longman Group, London, (1973).

95. M. Francon, Optical Interferometry (Academic Press, New York, 1966).

96. P. Jacquinot, Rep. Progr. Phys. 23, 268 (1960).

97. A. Cook, Interference of Electromagnetic Waves (Clarendon Press, Oxford, 1971), Chapter 6.

98. Burleigh Instruments, Inc., East Rochester, New York, Technical memo. No. FP 140475 (Revised April 1976).

99. M. Born and E. Wolf, Principles of Optics (Pergamon Press, Oxford, 1964), page 323. 
100. G. Fowles, Introduction to Modern Optics, 2nd Ed., (Holt, Rinehart and Winston, New York, 1975), Chapter 4.

101. P. Jacquinot and C. Dufour, J. Rech. Centre Nat. Rech. Sci. Lab. Bellevue (Pari.s) 6, l (1948).

102. J. Mack, D. McNutt, F. Roesler and R. Chabbal, Appl. Opt. $\underline{2}, 873$ (1963).

103. K. Kielenz, R. Stephens and K. Nefflen, J. Res. Nat. Bur. Stand. 68C, 1 (1964).

104. J. Greig and J. Cooper, Appl. Opt. 7, 2166 (1968).

105. RCA Publication PIT-700B, Dec. 1971, RCA Electronic Components Div., Harrison, N.J.

106. J. Cooper, The Minicomputer in the Laboratory (WileyInterscience, New York, 1977).

107. M. Pinta, Atomic Absorption Spectrometry (Hilger, Tondon, 1975), Châpter 4 .

108. V. Fasse1, W. Barnett and R. Kniseley, Spectrochim. Acta, Part B, 25, 139 (1970).

109. J. Fritz and G. Schenk, Jr., Quantitative Analytical Chemistry (Allyn and Bacon, Boston, 1974), Chapters 24 and 30 .

110. Target Values and Vial Codes for Series 200 Serum, $\mathrm{CH}$ 46-DA (Revised 12/74), Dade Div., American

Hospital Supply Corp., Miami, Florida.

111. Radiometer Model FLM3 Flame Photometer Technical Bulliten 32.137 (1976), Radiometer Corporation, Copenhagen, Denmark.

112. V. Fassel, R. Slack and R. Kniseley, Anal. Chem. 43, 186 (1971).

113. Software development performed by B. M: Golden at Ames Laboratory - USERDA under contract W-7405-eng-82 with the Atomic Energy Commission, September 1976.

114. PDP 11/10 Processor Handbook, Digital Equipment Corp., Maynard, Mass., 1973. 
115. RT Il System Reference Manual, Tech. Publ. DEC-11ORUGA-C-D, Digital Equipment Corp., Maynard, Mass., Revised, June 1975. 


\section{ACKNOWLEDGMENTS}

I wish to express my gratitude to Dr. Edward Yeung whose interest, cooperation and timely suggestions aided in the completion of this work and my graduate program, and to my parents for their encouragement throughout my entire education.

Thanks arc also due to Cris, Lester and Johnie for never letting me take myself too seriously and to Cecil who reminded me that occasionally I should.

Finally, my deepest gratitude goes to my wife, Nancy, and daughter Katie for their patience and forbearance during the past two years. 


\section{APPENDIX A: ELECTRONIC CIRCUITRY}

\section{A. Ramp Generator}

Figure 17 shows a schematic of the ramp generator. The circuit utilizos a 555 integrated circuit timer. Pins 6 and 7 are held at the same voltage as pin 1 until the potential at pin 2 falls below $1 / 3$ of the supply voltage $\left(V_{S}\right)$ at which point pins 6 and 7 are separated from pin 1 and the ramping process is begun. The .47 $\mathrm{ff}$ capacitor is linearly charged with the constant current provided by the 2 N3638 pnp transistor and IM potentiometer network. When the voltage at pin 6 is $2 / 3 \mathrm{~V}_{\mathrm{s}}$, pin 7 shorts to pin 1 and thus the capacitor is rapidly discharged. If the switch connected to pin 2 is in the single scan (open) position, the capacitor will discharge completely and will only be repeated when the pushbutton trigger switch is momentarily depressed. If the switch is in the repeat (closed) position, the capacitor discharges to $1 / 3 \mathrm{~V}_{\mathrm{s}}$ and begins to recharge again. When used as part of the computer interface, a positive going TTL pulse from the DAC momentarily turns on the 2N3643 npn transistor which in turn drops pin 2 below $1 / 3 \mathrm{~V}_{\mathrm{s}}$ and causes a ramp to begin. When operated in the single scan mode, the output is followed with a 741 operational amplifer in a unity gain configuration and when used with the computer this output is divided and buffered with a 


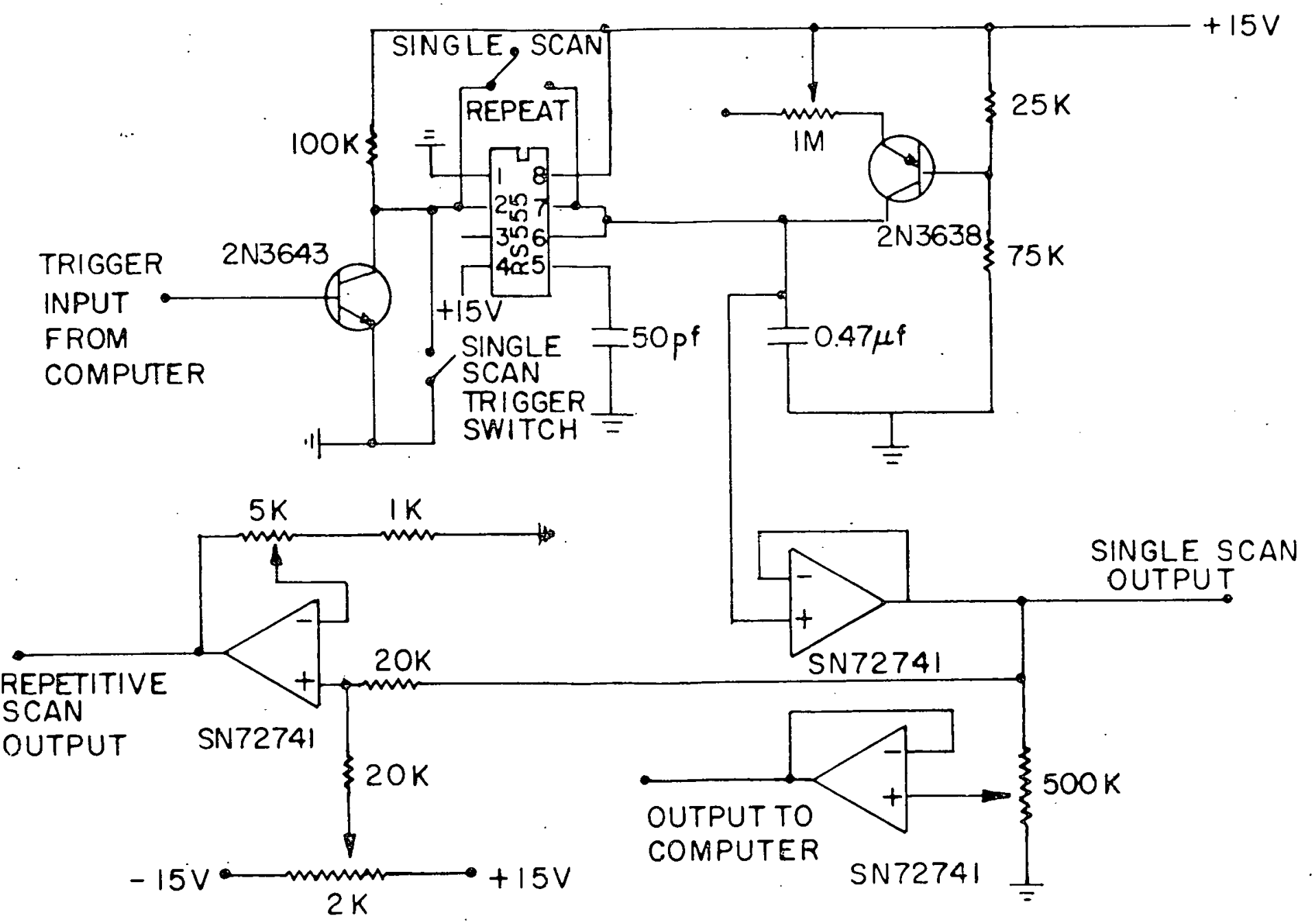

Figure 17. Circuit schematic of the linear ramp generator 
second 741 before input to the ADC (the maximum input voltage for the $A D C$ is $\pm 1 V)$. Since the output is from $1 / 3$ to $2 / 3 \mathrm{~V}_{\mathrm{S}}$ when operating in the repeat mode, it must be biased and amplified using a third 741 to again provide a ramp of 0 to $2 / 3 \mathrm{~V}_{\mathrm{S}}$. The entire circuit is powered by a Datel Model BPM 15/200 $\pm 15 \mathrm{~V}$ supply and is attached to a grey metal, rack mountable chassis.

\section{B. High Voltage Amplifier}

A circuit schematic for the high voltage amplifier used for driving the scanning crystal on the Fabry-Perot is shown in Figure 18a. As the 0 to $10 \mathrm{~V}$ ramp is input to the base of lle IJ423 npr transistor, the base to emitter voltage becomes more positive which turns on the transistor and causes a conventional current flow. This in turn causes the base of the S0028 pnp transistor to become more negative with respect to its emitter thus turning it on and allowing for the potential al the output to rise accordingly. By adjusting the potentiometer the gain may. be varied from 1 to 50. The circuit is powered by several high voltage batteries in series which produce a positive potential of $297 \mathrm{~V}$. Since the piezoelectric crystal is of such high impedance, the circuit consumes very little power and the batteries only need replacement at intervals of one year. 
(a)

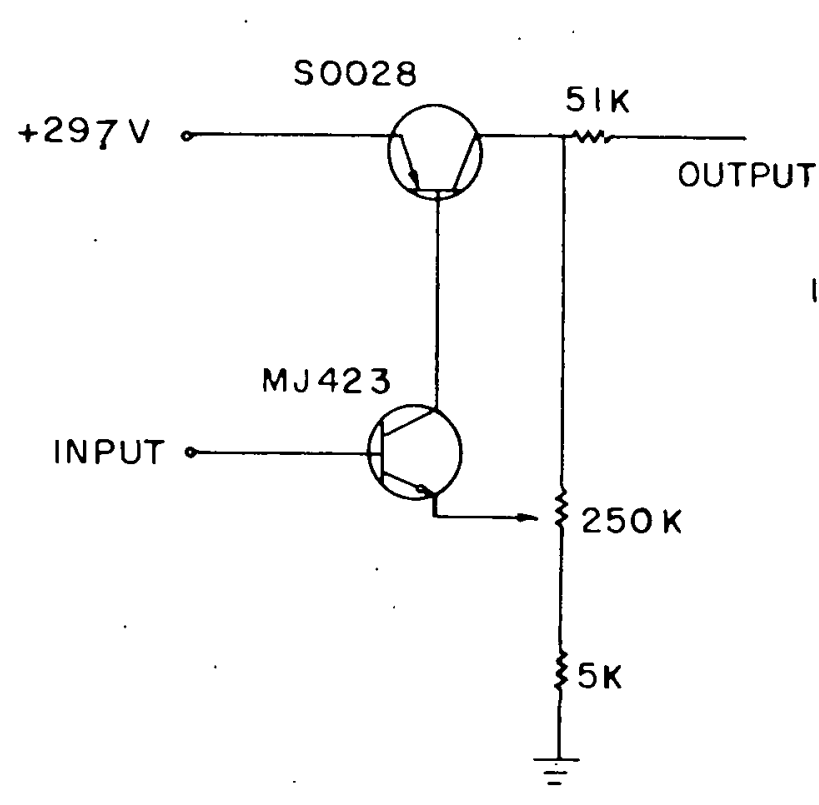

(b)

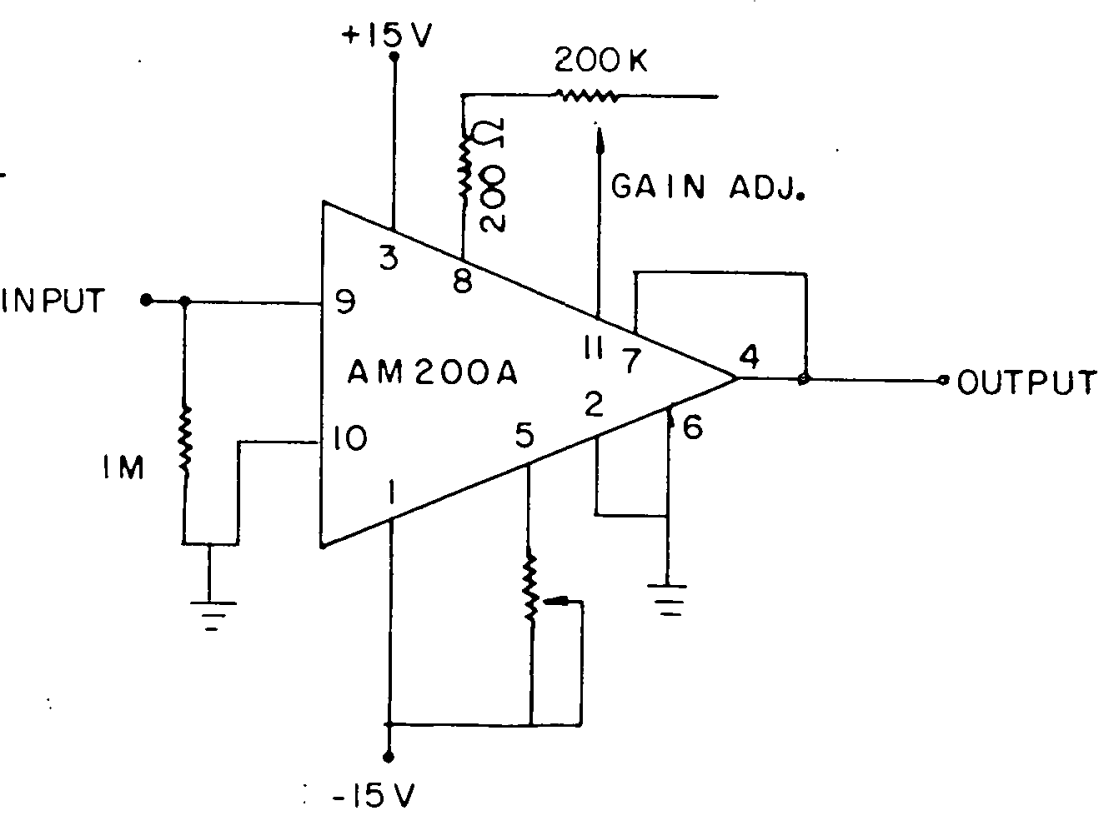

Figure 18. Circuit schematics for the high voltage amplifier (a) and buffer/ inverter (b) 


\section{Buffer/Inverter}

Because the photomultiplier is capable of producing currents in the $\mu \mathrm{A}$ range and the computer $\mathrm{ADC}$ has a low input impedance, a buffer is necessary between the PMT and the ADC. The schematic of this buffer circuit is shown in Figure 18b. It consists of a Datel Model AM200A instrumentation amplifier in an inverter with gain configuration. This amplifier features an input impedance of $10^{9} \Omega$ and is capable of providing an output current of $5 \mathrm{~mA}$. The gain is adjustable from 1 to 1000 using a 10 -turn $200 \mathrm{~K}$ potentiometer between pins 8 and 11 . The same Datel $\pm 15 \mathrm{~V}$ power supply utilized by the ramp generator is also used to power this circuit. Both the high voltage amplifier and the buffer/inverter are attached to a grey metal, rack-mountable chassis.

\section{Sample-Hold Detection System}

The schematic for one of the six parallel channels in the sample-hold detection system is shown in Figure 19. The ramp and a preset:voltage are the inputs to a LM311 comparator: When these two inputs are of the same potential, the comparator output undergoes a TTL low to high transition. The positive-going edge of this transition triggers a SN74121 one shot which in turn provides a TTL pulse of well 


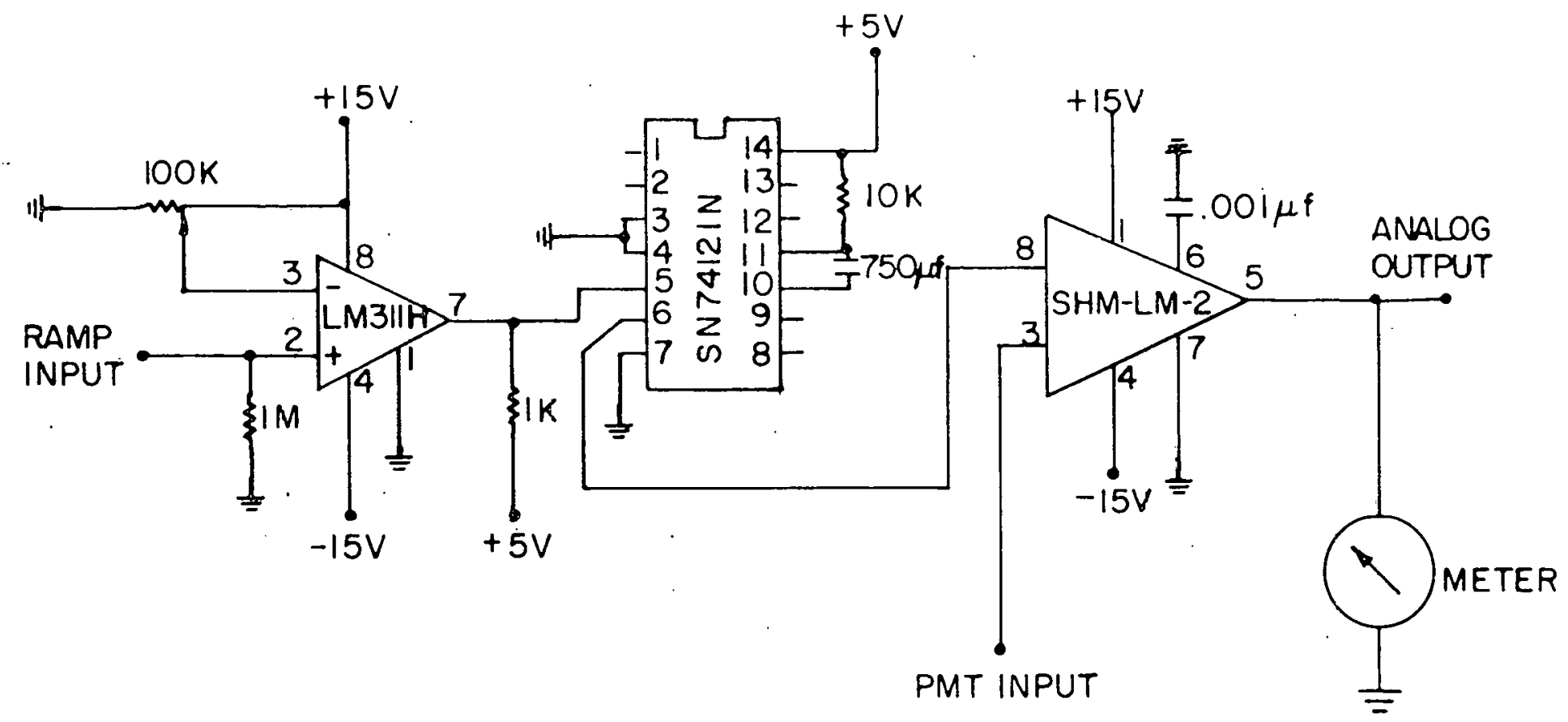

Figure 19. Circuit schematic for one channel of the six-channel sample-hold cirrcuit 
defined duration. In this case, the $10 \mathrm{~K}$ resistor and 750 pf capacitor provide a $5.2 \mathrm{\mu sec}$ output pulse which enables a Datel SHM-LM-2 sample-hold circuit for that amount of time. A .001 uf hold capacitor cormected to the SHM-LM-2 module allows the sampled voltage to be read up to 3 minutes after it is taken without appreciable bleed off. By adjusting the 10-turn $100 \mathrm{~K}$ potentiometer on the negative input of the LM3ll the position along the ramp (and hence the mirror spacing) at which the photomultiplier signal is read may be changed. 't'nis allows for location of peak maxima or changing to different emission peaks altogether. The voltage values recorded by each channel are read on either one of two voltmeters by rotating a selection switch to the desired channel. Datel BPM $15 / 200^{\circ} \pm 15 \mathrm{~V}$ and Zeltex 25-AX10005P +5V supplies are used to power all six channels and the entire circuit is attached to a single grey metal, rack-mountable chassis. 


\section{APPENDIX B: COMPUTER SOFTWARE}

The executive program for the Fabry-Perot interface is titled FABRY and is a FORTRAN routine that controls the entire operation of the interface. SAMP is an assembly language subroutine called by FABRY that initiates a scan and records the data from it in intermediate arrays. (ISAW, IMPT). Following the completion of each scan, the intermediate arrays are passed to FABRY which indexes the photomultiplier values according to the $\mathrm{X}$ (SAW) values and sums them to the proper position in a permanent $Y$ (PMT) array with the appropriate incrementation of the corresponding flag array (FLAG) position. This process is repeated until the required number of scans have been completed after which each PMT value is divided by the appropriate FLAG value to produce a weighted numerical average for each point. The PMT positions where FLAG $=0$ are sorted out and the resultant PMT array is ready for plotting or storage on the floppy disk. The graphics routines necessary for $X-Y$ plotting (ASCALE, LINE) were written and developed by Golden (113). All other call statements and functions used are part of the PDP-11 FORTRAN and MACRO software and are described in detail by the manufacturer $(114,115)$. 


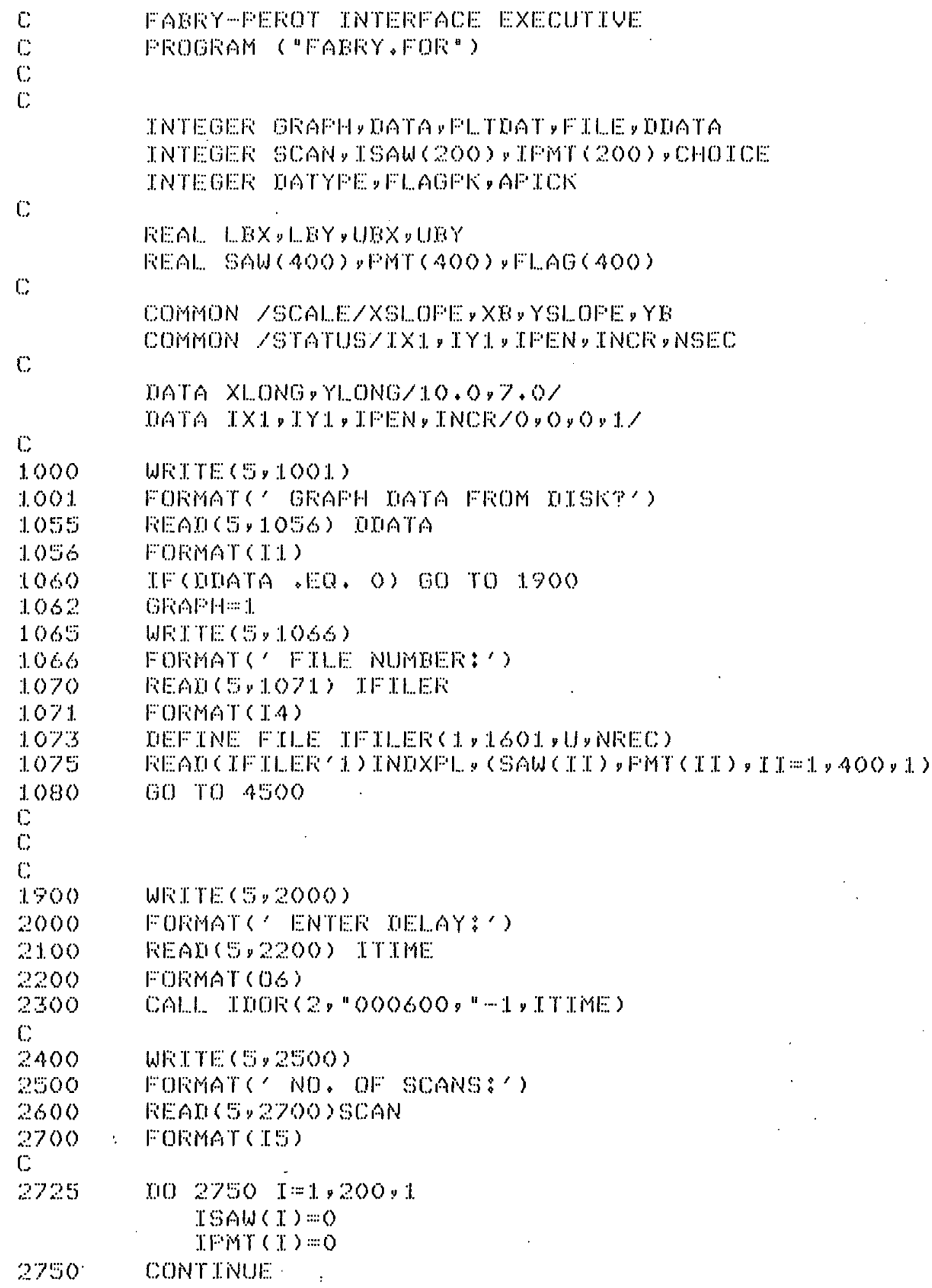




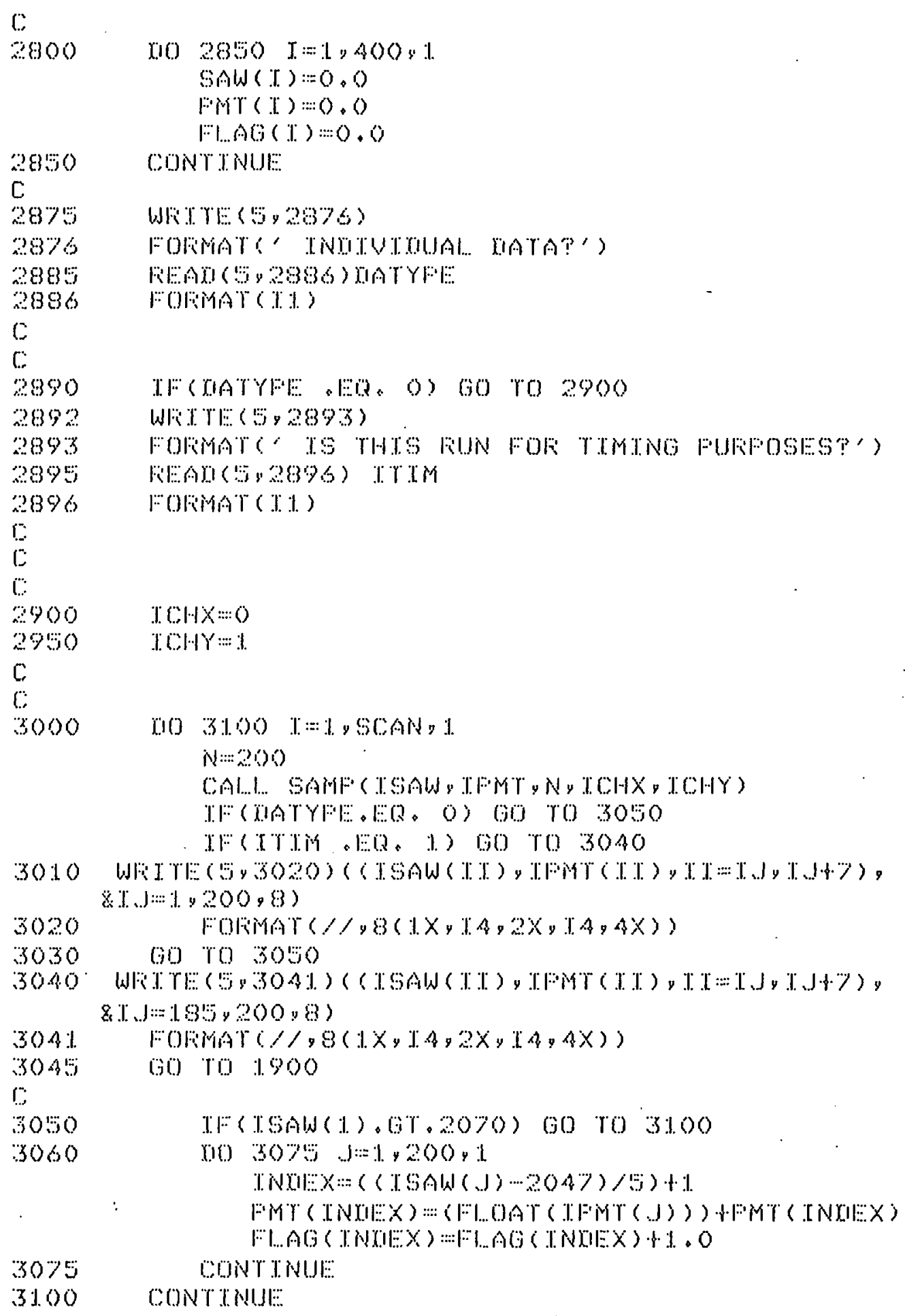




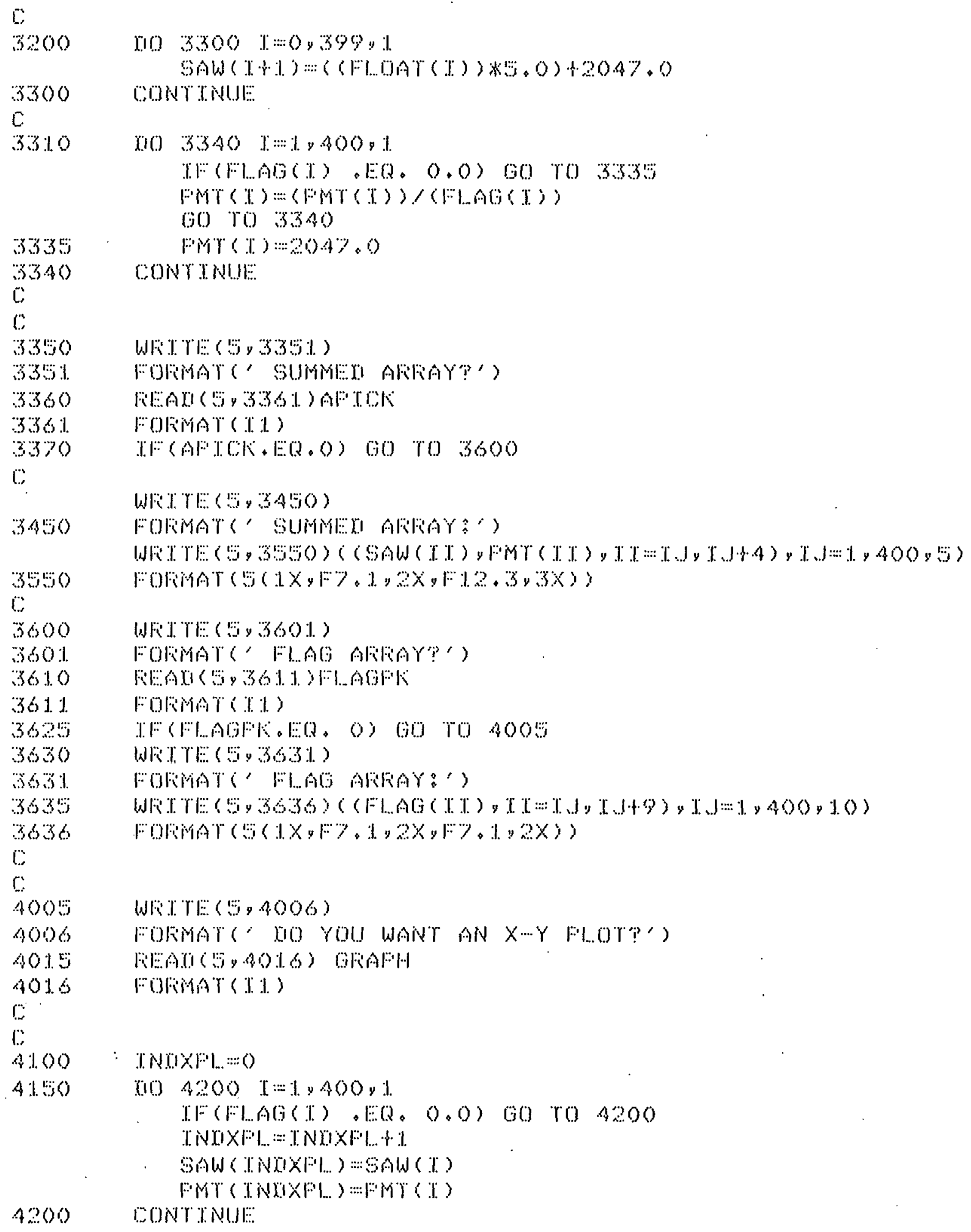




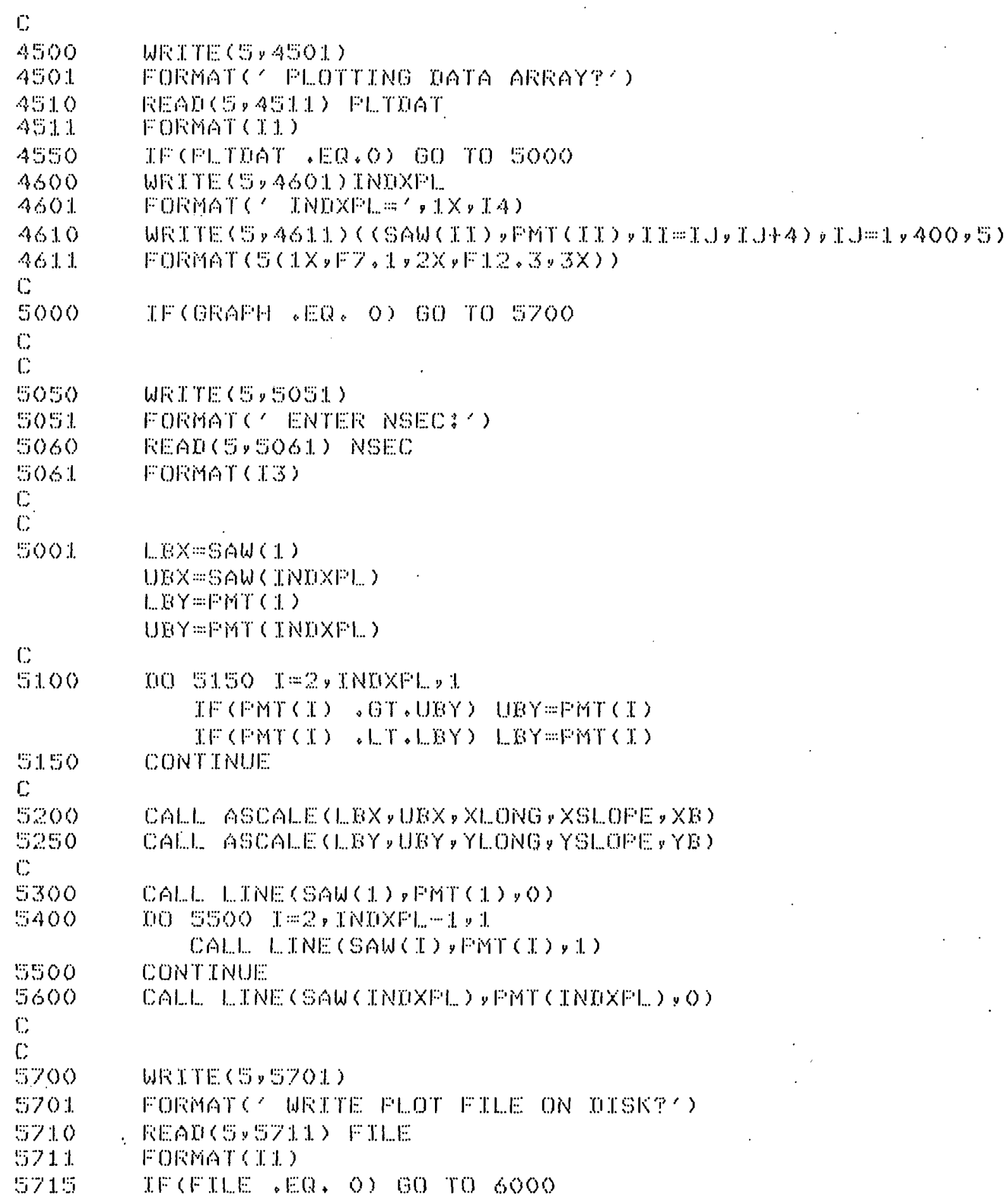




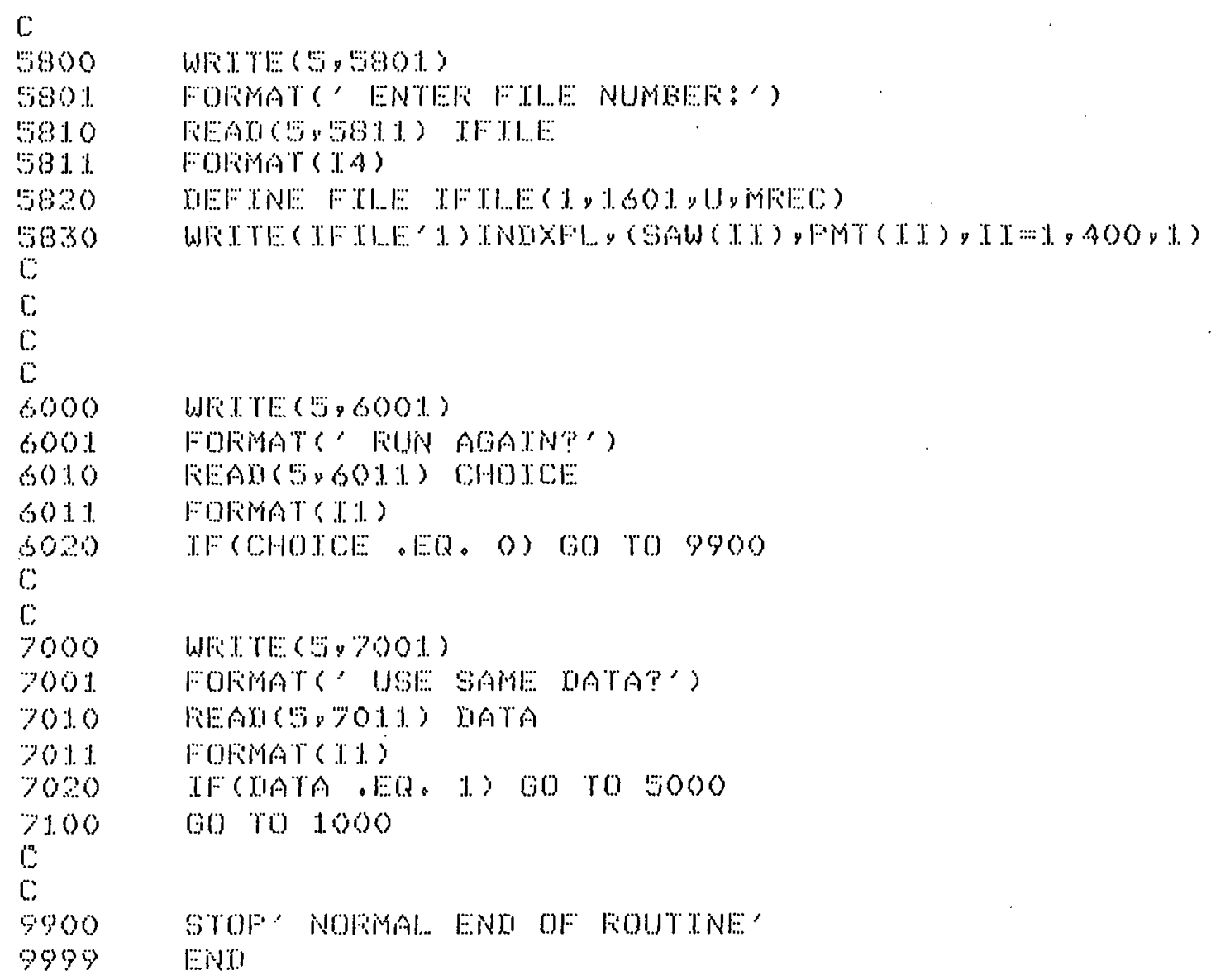




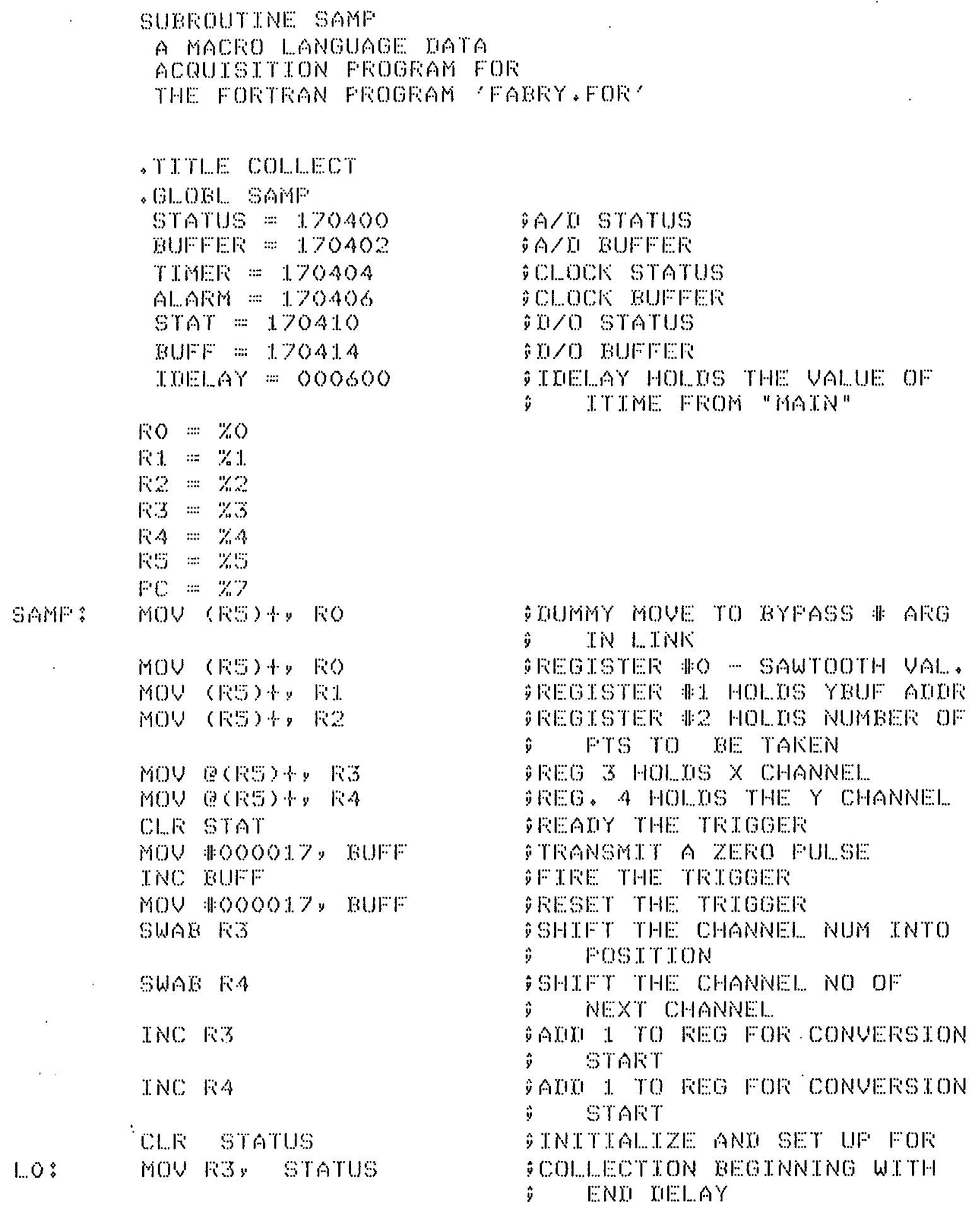




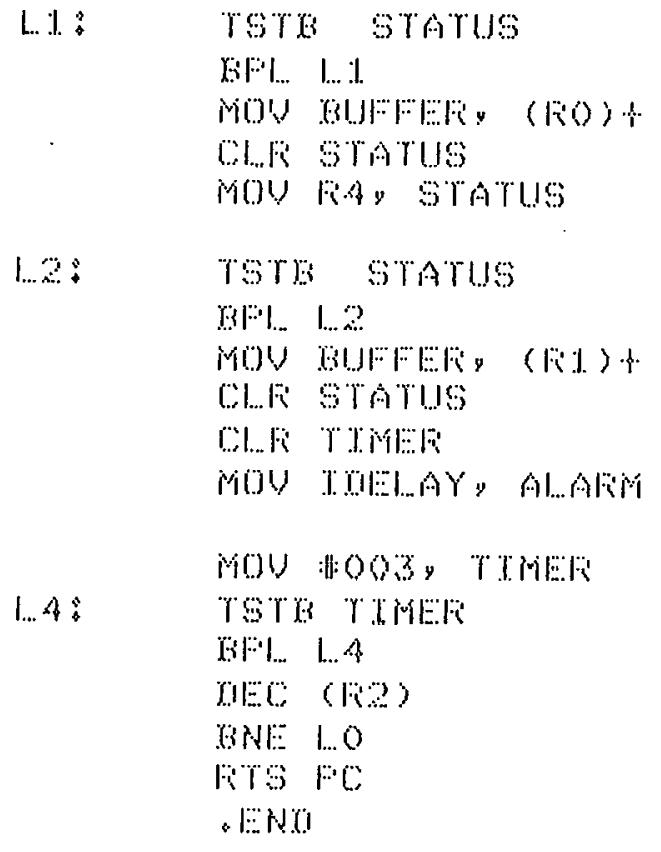

Y CHECK CONUERSTON STATUS

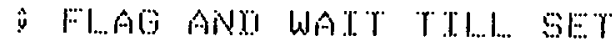

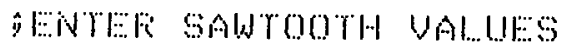

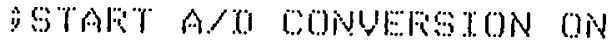
3) $r$ CHANNEI.

"CHEK TH CONVERSTON COMF. yे WATIT TILL OONE:

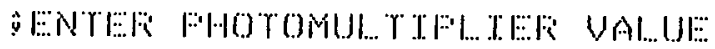

मेWEF TWE CIOCK

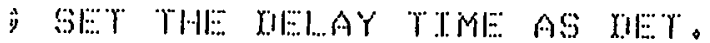

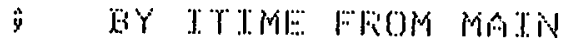

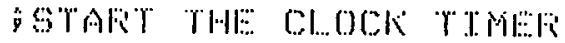

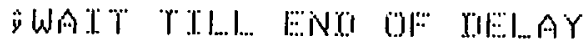

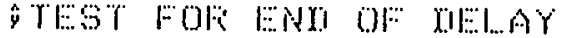

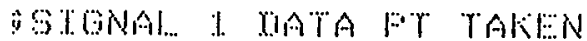

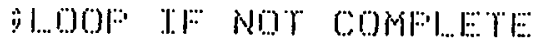

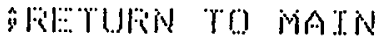

